\title{
An ADAR1-dependent RNA editing event in the cyclin-dependent kinase CDK13 promotes thyroid cancer hallmarks
}

Julia Ramírez-Moya ${ }^{1,2,3,4}$ (⿺辶, Christos Miliotis², Allison R. Baker², Richard I. Gregory ${ }^{3}$, Frank J. Slack ${ }^{2 *}$ and

Pilar Santisteban ${ }^{1,4^{*}}$ (1)

\begin{abstract}
Background: Adenosine deaminases acting on RNA (ADARs) modify many cellular RNAs by catalyzing the conversion of adenosine to inosine (A-to-I), and their deregulation is associated with several cancers. We recently showed that A-to-l editing is elevated in thyroid tumors and that ADAR1 is functionally important for thyroid cancer cell progression. The downstream effectors regulated or edited by ADAR1 and the significance of ADAR1 deregulation in thyroid cancer remain, however, poorly defined.
\end{abstract}

Methods: We performed whole transcriptome sequencing to determine the consequences of ADAR1 deregulation for global gene expression, RNA splicing and editing. The effects of gene silencing or RNA editing were investigated by analyzing cell viability, proliferation, invasion and subnuclear localization, and by protein and gene expression analysis.

Results: We report an oncogenic function for CDK13 in thyroid cancer and identify a new ADAR1-dependent RNA editing event that occurs in the coding region of its transcript. CDK13 was significantly over-edited (c.308A > G) in tumor samples and functional analysis revealed that this editing event promoted cancer cell hallmarks. Finally, we show that CDK13 editing increases the nucleolar abundance of the protein, and that this event might explain, at least partly, the global change in splicing produced by ADAR1 deregulation.

Conclusions: Overall, our data support A-to-l editing as an important pathway in cancer progression and highlight novel mechanisms that might be used therapeutically in thyroid and other cancers.

Keywords: ADAR1, A-to-l editing, Cancer, CDK13, Splicing

\footnotetext{
*Correspondence: psantisteban@iib.uam.es; fslack@bidmc.harvard.edu ${ }^{1}$ Instituto, de Investigaciones Biomédicas "Alberto Sols"; Consejo Superior de Investigaciones Científicas (CSIC), Universidad Autónoma de Madrid (UAM), Madrid, Spain

2 Department of Pathology, Harvard Medical School Initiative for RNA Medicine, Beth Israel Deaconess Medical Center, Harvard Medical School, Boston, MA, USA

Full list of author information is available at the end of the article
}

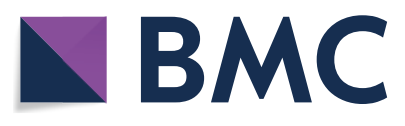

(S) 2021. Open Access This article is licensed under a Creative Commons Attribution 4.0 International License, which permits use, sharing, adaptation, distribution and reproduction in any medium or format, as long as you give appropriate credit to the original author(s) and the source, provide a link to the Creative Commons licence, and indicate if changes were made. The images or other third party material in this article are included in the article's Creative Commons licence, unless indicated otherwise in a credit line to the material. If material is not included in the article's Creative Commons licence and your intended use is not permitted by statutory regulation or exceeds the permitted use, you will need to obtain permission directly from the copyright holder. To view a copy of this licence, visit http://creativecommons.org/licenses/by/4.0/. The Creative Commons Public Domain Dedication waiver (http://creativeco mmons.org/publicdomain/zero/1.0/) applies to the data made available in this article, unless otherwise stated in a credit line to the data. 


\section{Introduction}

Adenosine deaminases acting on RNA (ADARs) are involved in the most common type of RNA editing in mammals, A-to-I RNA editing [1]. Acting specifically on double-stranded RNAs, ADARs catalyze the post-transcriptional conversion of adenosine residues to inosines, which are recognized by the cellular machinery as guanosines. Accordingly, A-to-I editing can create, delete or alter the specificity of a codon or a splice site, alter RNA structures, or modify regulatory RNAs [1]. Importantly, when this occurs in coding regions it can lead to the generation of new proteins with novel functions [2]. As A-to-I RNA editing overlaps temporally and spatially with pre-mRNA splicing, it is likely that extensive crosstalk exists between the two processes. Indeed, A-to-I RNA editing has been shown to globally affect alternative splicing $[3,4]$; however, only a limited number of editing sites directly affect splice sites, and it is highly possible that additional mechanisms exist to regulate splicing $[3,4]$. Although poorly understood, RNA editing events within transcripts of genes related to splicing regulation might explain the global changes in splicing induced by the ADARs. ADAR1, which is expressed through two isoforms (p110 and p150) [2], is the most abundant member of the ADAR family of enzymes and plays important roles in both physiological and pathological processes.

RNA editing drives molecular diversity, offering an organism the potential for greater complexity. ADAR editing is essential for survival in mammals, but its deregulation is also associated with cancer initiation and progression $[5,6]$. Recent studies, especially those by The Cancer Genome Atlas (TCGA) project, have established that A-to-I editing levels and the enzymes mediating this modification are significantly altered in cancer $[7,8]$. Most tumor types have elevated levels of A-to-I editing and ADAR1 expression, and this latter finding has been associated with a reduction in patient survival in glioma, papillary thyroid and uterine corpus endometrial carcinomas [9]. The available data also suggest that edited RNAs might serve as novel candidates for therapeutic and diagnostic purposes [8]. In this context, we recently demonstrated the importance of A-to-I editing in thyroid cancer [10], where ADAR1dependent activity is markedly higher in tumors than in normal thyroid tissue $[7,8]$. Thyroid cancer is the most common endocrine malignancy [11], and its incidence has increased significantly in the US and in other countries over the last few decades [12-14]. Although it generally has a good outcome, the rate of disease recurrence is high, which is associated with increased incurability and with patient morbidity and mortality [15].
Indeed, some patients develop aggressive forms of the disease that are untreatable, and the molecular foundations remain poorly understood [15]. Accordingly, a better understanding of thyroid cancer is essential to develop new strategies and provide new therapeutics for treatment. Thyroid cancer encompasses several histological types and subtypes with different cellular origins, characteristics and prognoses. Most tumors originate from follicular cells and can be classified as well-differentiated carcinomas, including papillary (PTC) and follicular (FTC) thyroid carcinoma, whereas others are classified as poorly differentiated (PDTC) and undifferentiated or anaplastic (ATC) thyroid carcinomas $[16,17]$. The latter, less-differentiated, thyroid cancers (PDTC and ATC) can develop de novo, although many arise through the process of stepwise differentiation of PTC and FTC [17].

We previously demonstrated a functional role for ADAR1 and RNA editing in thyroid cancer tumorigenesis following ADAR1 gene silencing and pharmacological inhibition of ADAR1 editase activity. We also found that some microRNAs, such as miR-200b, are new targets for ADAR1 in thyroid cancer [10]. Still, several issues remain unresolved regarding how RNA editing affects thyroid cancer. In the present study, we used bioinformatic approaches and high throughput RNA-sequencing (RNA-seq) of ADAR1 knockdown cancer cells to globally examine how ADAR1 and its A-to-I RNA editing activity influences gene expression and mRNA splicing. This analysis allowed us to identify novel editing sites for ADAR1 in the transcriptome and uncover a new ADAR1-dependent RNA editing event that occurs in CDK13, which encodes a cyclindependent kinase implicated in the regulation of transcription [18] and RNA splicing [19, 20]. We found that the nucleotide modification from this editing event is overrepresented in thyroid tumors and has functional consequences in thyroid cancer cells. Overall, our data point to an oncogenic role for $C D K 13$ in thyroid cancer, as the ADAR1-dependent editing of $C D K 13$ provides an advantage for cancer progression and may explain the global change in splicing pattern observed upon ADAR1 knockdown.

\section{Materials and methods \\ Patients}

Samples of paired PTC tumors and contralateral normal thyroid tissue from patients $(n=6)$ were obtained from the Biobank of the La Paz University Hospital (Madrid, Spain). The main clinical characteristics of the patients have been described [10]. Informed consent was obtained from all the patients following the protocols approved by the hospital ethics committee. 


\section{Cell culture}

Cal62 and TPC1 tumor thyroid cell lines were grown in Dulbecco's modified Eagle's medium (DMEM) supplemented with $10 \%$ fetal bovine serum (FBS). The Cal62 cell line was obtained from Leibniz-Institut DSMZ-German Collection of Microorganisms and Cell Cultures and the TPC1 cell line was provided by Dr. A.P. Dackiw (Johns Hopkins University, Baltimore). Cells were tested for mycoplasma contamination and authenticated every 6 months by short tandem repeat profiling using the Applied Biosystems Identifier kit in the Genomic Facility at the Institute of Biomedical Research (IIBm, Madrid, Spain).

\section{siRNAs, plasmids and transfections}

The following siRNAs were purchased from Thermo Fisher Scientific Inc. (Waltham, MA): siControl (Silencer Select Negative Control \#1 4,390,843), siADAR1 \#1 (s1007), siADAR1 \#2 (119,581), siCDK13 \#1 (s16397), siCDK13 \#2 (s16398), siCDK13 \#3 (s16399). All siRNA were transfected using Lipofectamine RNAimax (Thermo Fisher Scientific). Lentiviral plasmids expressing the wild-type (WT) CDK13, the c.308A > G edited (edit) form or the empty plasmid were designed and constructed by VectorBuilder Inc. (Chicago, IL). Puromycin was used for selection.

\section{RNA sequencing}

Total RNA was isolated from transfected cells using the mirVana Isolation Kit (Ambion, Austin, TX) and was processed at the Dana Farber Molecular Biology Core Facility (two biological independent replicates). RNA quality was confirmed on the Agilent Bioanalyzer (Santa Clara, CA). Library preparation was performed using the KAPA mRNA HyperPrep Kit for poly $(\mathrm{A}+$ ) (Roche; Basel, Switzerland). RNA-seq analysis was performed on the Illumina NextSeq 500 platform (San Diego, CA) using 75-bp paired-end reads. The data have been deposited in the NCBI Gene Expression Omnibus [21] and are accessible through the GEO Series accession number GSE165282 (https://www.ncbi.nlm.nih.gov/geo/query/ acc.cgi?acc $=$ GSE165282).

\section{Differential expression analysis}

Differential expression comparisons were performed for siControl versus siADAR\#1 and siADAR\#2. All samples were processed using an RNA-seq pipeline implemented in the bcbio-nextgen project (https://bcbio-nextgen. readthedocs.org). Raw reads were examined for quality issues using FastQC (http://www.bioinformatics.babra ham.ac.uk/projects/fastqc/) to ensure that library generation and sequencing were suitable for further analysis. Adapter sequences and other contaminant sequences were trimmed from reads using Atropos [22]. Counts of reads aligning to known genes were generated by featureCounts [23]. In parallel, Transcripts Per Million (TPM) measurements per isoform were generated by quasialignment using the Salmon tool [24]. Normalization at the gene level was called with DESeq2 [23, 25], with preference to use counts per gene estimated from the Salmon quasialignments by Tximport [23, 25, 26]. The DEGreport Bioconductor package was used for quality control and clustering analysis (https://doi.org/10.18129/B9.bioc. DEGreport). DESeq2 was used for differential expression analysis.

\section{Variant calling analysis}

BAM files were processed with GATK [27] following the best-practices for RNA-seq variant calling, to compile a list of nucleotide variants in each sample. In addition, we added an additional filter to remove calls within 10 bases of a junction on either side. Variants were annotated with the SnpEff tool [28]. For differential allele frequency analysis, we removed all annotated single nucleotide polymorphisms (SNPs), and fitted a linear model to the allele frequency values from the two groups: siADAR1 $\# 1 / 2$ and siControl. We employed the Benjamin-Horchberg method for $\mathrm{p}$-value correction to deal with multiple testing.

\section{Splicing analysis}

Differential splicing analysis was performed using Multivariate Analysis of Transcript Splicing (rMATS) (http://rnaseq-mats.sourceforge.net/) with default parameters. The RNA-seq reads were mapped to the human genome assembly GRCh38 using the STAR aligner. rMATS evaluates splicing per sample in two ways: by counting only the number of reads that map to the splice junctions (JC analysis), and by also counting the reads that map within the alternately spliced target region (JCEC analysis). The JCEC output was used for further analysis. Differential splice comparisons were performed for both siControl versus siADAR\#1 and siControl versus siADAR\#2. Inclusion and exclusion junction reads were averaged from replicates and used to calculate the Inclusion Level Difference (PSI score) for each splice site. Hits were filtered by removing sites with $<15$ reads total in either sample average (siControl or siADAR1) and by using a false discovery rate (FDR) cut-off of $<0.05$.

\section{Functional annotation of candidate genes}

The genes obtained after the RNA-seq analysis were processed by The Database for Annotation, Visualization and Integrated Discovery (DAVID, http://david.abcc.ncifcrf. gov) for functional annotation. 


\section{RNA quantification}

For gene expression analysis, total RNA was isolated with Trizol Reagent (Invitrogen). Template cDNA synthesis was performed using the NZY M-MuLV First-Strand cDNA Synthesis Kit (Nzytech, Lisbon, Portugal). Quantitative reverse transcription-PCR (qRT-PCR) was performed with the KAPA SYBR Fast Universal Kit from Sigma-Aldrich. (Madrid, Spain) All primers were purchased from Sigma-Aldrich and are described in Table S1.

\section{Verification of RNA editing sites}

The RNA editing site c.308A $>$ G was verified using RTPCR with the following PCR primers: forward primer: 5'-CTCTGGAGGTCAAGCGGC-3' and reverse primer: 5'-GACTGGGAGCTCACATCCTC-3'. PCR products were evaluated by Sanger sequencing and editing levels were calculated with INDIGO (https://www.gear-genom ics.com/indigo/).

\section{Protein extraction and western blotting}

Cells were lysed and proteins extracted with RIPA buffer [29]. Protein concentration was measured using the Bradford method (Bio-Rad Laboratories, Hercules, CA). Samples were separated by sodium dodecyl sulfate-polyacrylamide gel electrophoresis (SDS-PAGE) and transferred to nitrocellulose membranes (Bio-Rad). Immunoreactive proteins were visualized by enhanced chemiluminescence (GE Healthcare Bioscience, Pittsburgh, PA).

\section{Immunofluorescence}

Cells were seeded on coverslips for $48 \mathrm{~h}$, washed with phosphate-buffered saline (PBS), fixed in $4 \%$ paraformaldehyde for $15 \mathrm{~min}$, permeabilized with PBS containing $0.1 \%$ NP40 for $10 \mathrm{~min}$, washed again and then blocked at room temperature with PBS-5\% Tween containing 3\% bovine serum albumin and 1:100 normal goat serum for $30 \mathrm{~min}$. Coverslips were incubated with a 1:100 dilution of primary antibodies in blocking solution overnight at $4{ }^{\circ} \mathrm{C}$, washed three times in PBS-5\% Tween for $5 \mathrm{~min}$ and incubated for $1 \mathrm{~h}$ at room temperature with the secondary antibodies (Jackson Immunoresearch, West Grove, PA). After rinsing three times with PBS-5\% Tween, with the last wash containing 1:5,000 4,6-diamidino-2-phenylindole (DAPI), cells were finally mounted on Prolong antifade reagent (Invitrogen, Carlsbad, CA). Cells were observed using a confocal microscope with $\times 63$ oil immersion objective; $\times 2$ zoom was used for Cal62 cells. Colocalization was analyzed using the Fiji-Coloc2 plugin.

\section{Antibodies}

The following antibodies were obtained from Santa Cruz Biotechnology Inc. (Santa Cruz, CA): SP1 (sc-17824), tubulin (sc-5286) and vinculin (sc-73614). CDK13 (ab251955) and Fibrillarin (ab4566) antibodies were purchased from Abcam (Cambridge, UK). The GAPDH (MAB374) antibody was from EMD Millipore Corp. (Billerica, MA) and the hemagglutinin (HA) antibody (C29F4) was from Cell Signaling Technologies, (Danvers, MA).

\section{Proliferation and cell viability assays}

Cell proliferation was measured by DNA synthesis using bromodeoxyuridine (BrdU) labeling with an assay from Sigma-Aldrich (\#11,669,915,001). Briefly, cells were seeded in 96-well plates, pulse-labeled for $5 \mathrm{~h}$ with $10 \mu \mathrm{M}$ BrdU, and BrdU incorporation was measured in a luminometer (Promega, Madison, WI) [30]. Cell viability was determined using an XTT metabolic assay (Canvax Biotech, Córdoba, Spain) and crystal violet staining. For XTT analysis, cells were seeded in 96-well plates and dye reduction was recorded on a spectrophotometer after 72 h. For crystal violet staining, Cal62 and TPC1 cells were seeded in each well of a 6-well plate. Individual wells were fixed in $4 \%$ paraformaldehyde after 7-10 days and stained with crystal violet. After extensive washing and drying, the staining reagent was resolubilized in $1 \%$ acetic acid and quantified at $590 \mathrm{~nm}$ as an indirect measure of cell number.

\section{Invasion assay}

Cell invasion was assayed in Transwell cell culture chamber filters coated on the upper side with Matrigel (Corning BioCoat, Corning, NY), as described [29, 30]. Briefly, 45,000 Cal62 cells or 50,000 TPC1 cells in DMEM containing $0.2 \%$ FBS were seeded in the upper chamber and $20 \%$ FBS was added to the bottom chamber as a chemoattractant. Cells were allowed to invade for $22 \mathrm{~h}$. For the analysis of cell invasion using the CDK13-WT and -EDIT constructs, a total of 35,000 Cal62 and 15,000 TPC1 cells were used.

\section{Results \\ Consequences of ADAR1 knockdown on global gene expression}

We previously demonstrated a key role for $A D A R 1$ in thyroid tumorigenesis [10]. Loss-of-function of ADAR1 profoundly repressed proliferation, invasion, and migration in human thyroid tumor cell models and inhibited tumor growth in an in vivo xenograft model. Encouraged by these results, here we further investigated the function of $A D A R 1$ in thyroid cancer by examining the consequences of ADAR1 knockdown on global gene expression. We performed RNA-seq in Cal62 thyroid cancer cells and compared the mRNA expression of siControl cells (transfected with a control siRNA) with 


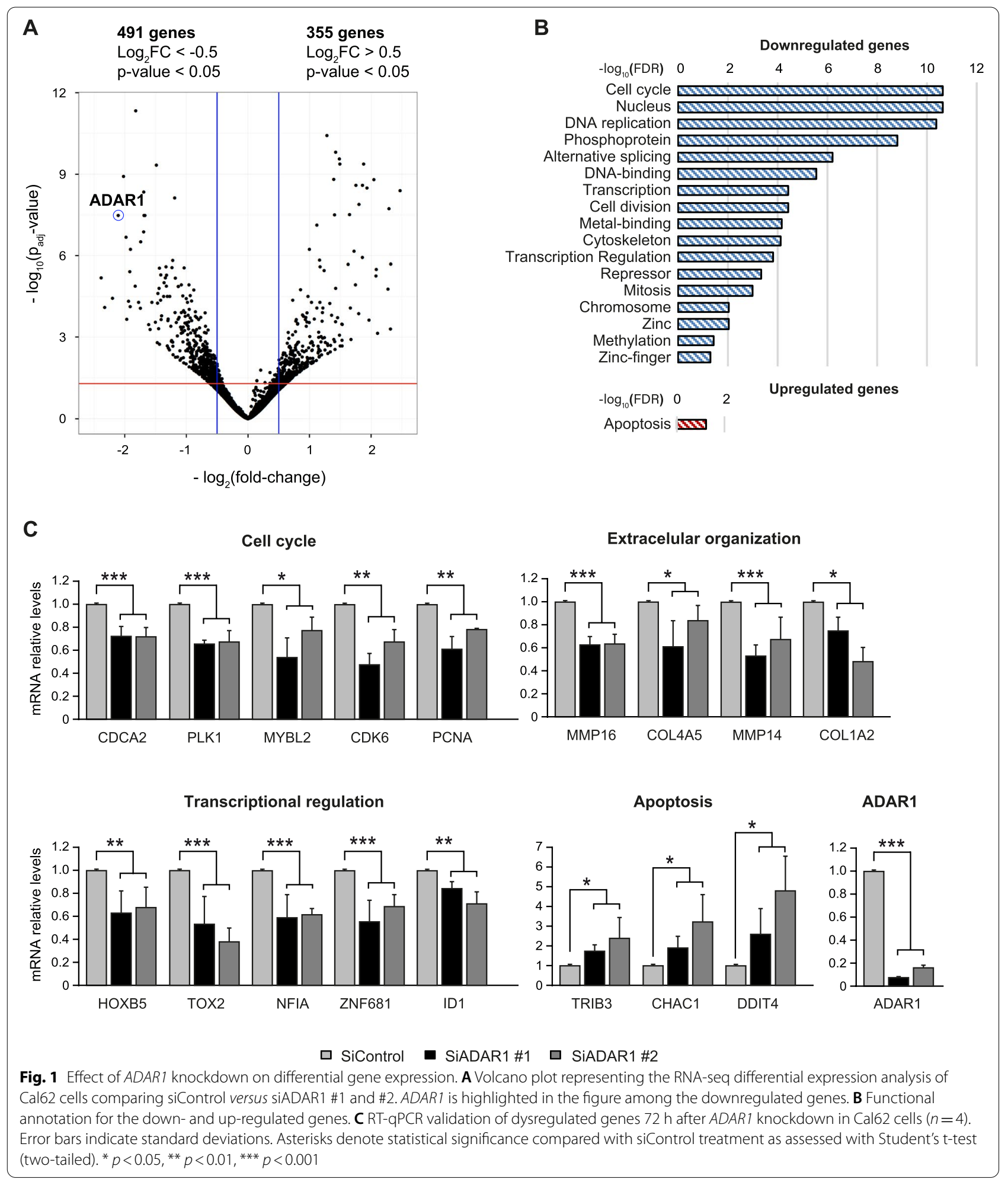

those silenced for $A D A R 1$ using two independent siRNAs (ADAR1 \#1 and ADAR1 \#2). Of note, the two siRNAs employed targeted both the p150 and p110 ADAR1 gene forms (Supplementary Figure 1).
We considered significant differences as those with an FDR-adjusted p-value $<0.05$ and a $\log _{2}$ Fold Change $(\mathrm{FC})<-0.5$ for the downregulated events and a $\log _{2} \mathrm{FC}>0.5$ for the upregulated events. We found 
that 491 genes were downregulated and 355 genes were upregulated upon $A D A R 1$ knockdown (Fig. 1A). Reassuringly, $A D A R 1$ emerged as one of the main downregulated genes, confirming its knockdown in these cells (Fig. 1A). Functional annotation of the deregulated genes showed an enrichment of genes involved in cell cycle, DNA replication, cell division and cytoskeleton among the downregulated genes, and genes involved in apoptosis among the upregulated genes (Fig. 1B). These results fit well with the reduced proliferation, invasion and cell viability after $A D A R 1$ silencing in thyroid cancer models [10]. Some studies have previously implicated ADAR1 in alternative splicing regulation [3, 4] and, notably, genes involved in the alternative splicing process were well represented in our downregulated data set (Fig. 1B). Finally, genes associated with transcription and regulation were also enriched in the downregulated data set (Fig. 1B). We validated some of the RNA-seq results by qPCR in ADAR1knockdown Cal62 cells; specifically, the highly significant candidate genes related to the aforementioned processes of cell cycle, extracellular organization, transcription regulation and apoptosis (Fig. 1C).

\section{ADAR1 has a major influence on global splicing patterns}

Given our results and the link between A-to-I RNA editing of pre-mRNAs by ADARs and splicing regulation [3, 4], we aimed to examine how ADAR1 editing globally influences alternative splicing. Several types of alternative splicing have been experimentally described [31], including skipped exons (SE), retained introns (RI) in the mature mRNA, mutually exclusive exons (MXE) and alternative 5' or 3' splice sites (A5SS or A3SS). We used the rMATS tool to determine differential alternative splicing [32] and filtered out events with $<15$ total junction reads. We considered as significant events those with an FDR-adjusted p-value $<0.05$ and an Inclusion Level Difference $<-0.2$ for downregulated events and $>0.2$ for upregulated events. This analysis revealed 1075 highconfidence differential splicing events when comparing the siControl cells and the $A D A R 1$-silenced cells using siRNA \#1, and 776 high-confidence differential splicing events with the siRNA \#2, with 114 differential alternative splicing events common in both comparisons
(Fig. 2A). These results suggest that $A D A R 1$ silencing has an influence on the global splicing pattern of thyroid cancer cells. Among the alternative splicing types observed in the 114 common events, the majority were SE (85.96\%) followed by A5SS (5.26\%), RI (3.51\%), MXE (2.63\%) and A3SS (2.63\%) (Fig. 2B). Regarding the affected splicing events, their inclusion was either repressed or promoted by ADAR1, although a general higher inclusion was observed in siADAR1 cells (data not shown). Functional annotation of the common alternately spliced genes revealed that the enriched genes were mainly involved in alternative splicing itself and in cytoskeleton processes (Fig. 2C). This latter finding may explain, at least in part, the suppression of migration and invasion observed in ADAR1-silenced cells [10]. As before, we experimentally validated the alternative splicing of selected candidate genes involved in cytoskeleton regulation by qPCR assays designed to distinguish between the spliced transcripts (Fig. 2D).

\section{ADAR1 edits hundreds of transcripts in thyroid cancer cells} We next analyzed the editing profile under the same conditions. A-to-I editing occurs at the level of RNA, but when the RNA is reverse transcribed to complementary DNA the inosine is converted to guanine. This A-to-G conversion, which is evidence of RNA editing, can be identified by comparing cDNA sequences with the reference genome. Global single nucleotide variant analysis revealed an enrichment in $\mathrm{A}>\mathrm{G}$ or complementary $\mathrm{T}>\mathrm{C}$ changes in $A D A R 1$-silenced cells, which is to be expected since ADAR1 is responsible for A-to-I editing (Fig. 3A). Moreover, the total number of variants was dramatically repressed by silencing of $A D A R 1$ (Fig. 3B). These data confirm that $A D A R 1$ knockdown reduces global editing activity. After SNP filtering, 122 A-to-I editing events $(F D R<0.05)$ were found in the transcriptome of the siControl cells and were markedly lower in siADAR1 \#1 and \#2 cells (FC >0). Based on location, the RNA editing sites could be divided into different categories: 3' untranslated region (UTR), 5'UTR, upstream and downstream gene regions, missense, nonsense or synonymous. We identified 113 RNA editing sites in 3'UTRs, 6 in downstream and 2 in upstream gene regions, and 1

\footnotetext{
(See figure on next page.)

Fig. 2 Consequences of $A D A R 1$ knockdown on alternative splicing. A Venn diagram representing the significant (FDR $<0.05$ and Inclusion Level Difference $<-0.2 /<0.2$ ) alternative splicing events differing between siControl and siADAR1 \#1 (blue) or siADAR1 \#2 (orange) in Cal62 cells. One hundred and fourteen events were common in both analyses. B Left panel: Schematic of the alternative splicing events analyzed. Right panel: Pie chart of events with significantly different inclusion levels (FDR $<0.05$ and Inclusion Level Difference $<-0.2$ / $<0.2)$ upon $A D A R 1$ knockdown. Abbreviations: SE, skipped exon; RI, retained intron; MXE, mutually exclusive; A5SS, alternative 5' splice site; A3SS, alternative 3'splice site. C Functional annotation for the genes that present with significant differential alternative splicing events after ADAR1 silencing. D RT-qPCR validation of ADAR1-regulated alternative splicing events for indicated genes $72 \mathrm{~h}$ after siRNA transfection in Cal62 cells ( $n=4$ ). Error bars indicate standard deviations. Asterisks denote statistical significance compared with siControl treatment as assessed with Student's t-test (two-tailed). ${ }^{*} p<0.05$, ** $p<0.01,{ }^{* * *} p<0.001$
} 
A

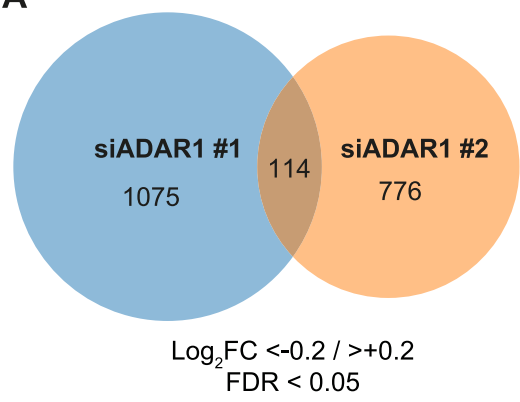

B

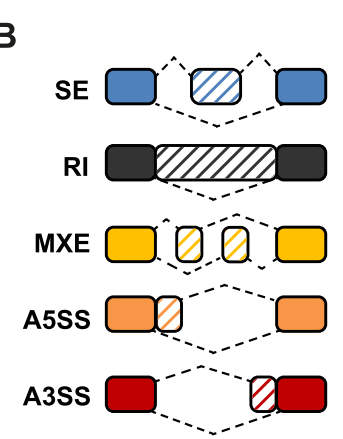

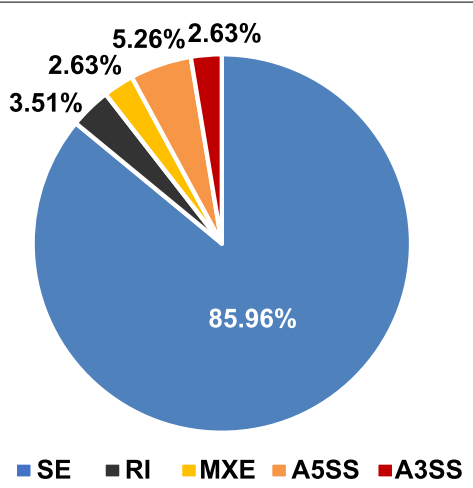

C $\begin{array}{llllllllll}-\log _{10}(\mathrm{FDR}) & 0 & 1 & 2 & 3 & 4 & 5 & 6 & 7 & 8\end{array}$

Alternative splicing

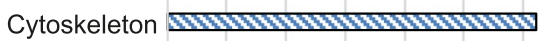

Phosphoprotein

Cytoplasm

Coiled coil

D

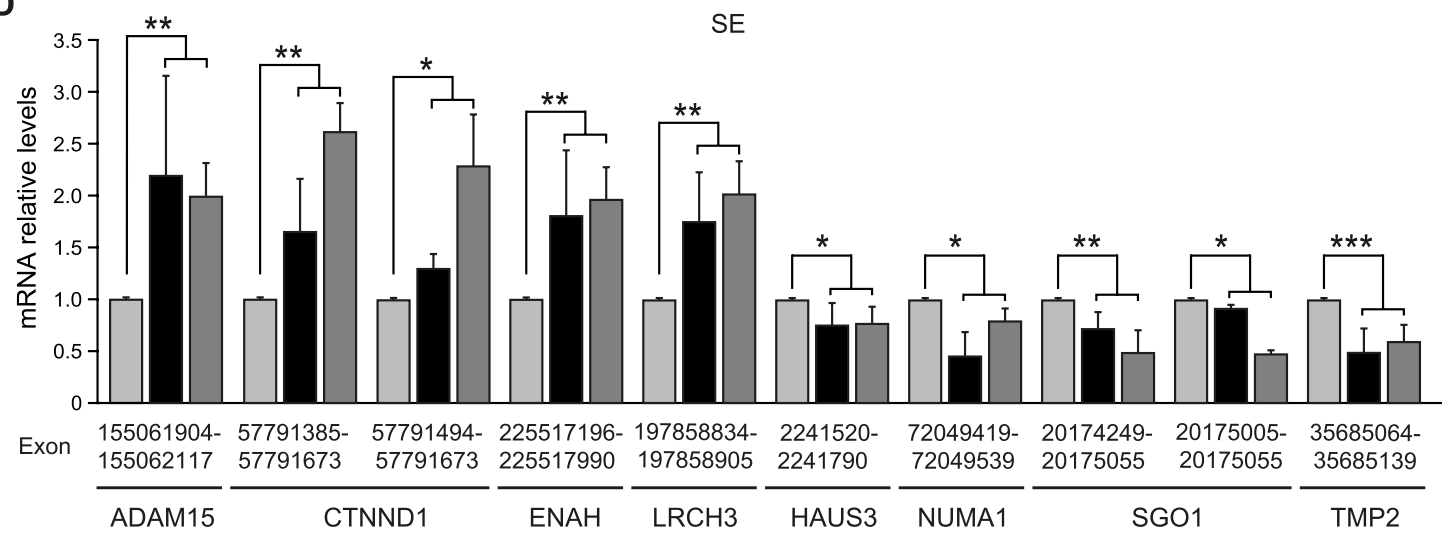

A5SS

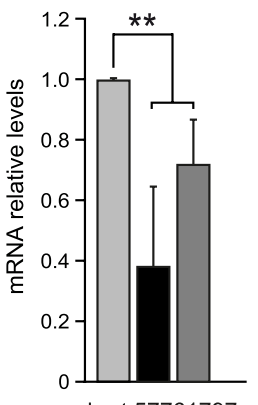

Exon short 57761797

$$
\frac{57762046}{\text { CTNND1 }}
$$

MXE

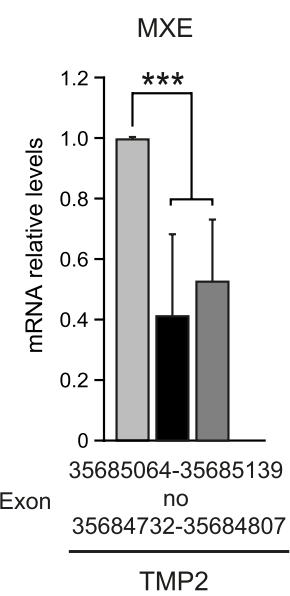

口 SiControl

- SiADAR1 \#1

口 SiADAR1 \#2

Fig. 2 (See legend on previous page.) 
missense variant (Fig. 3C). Notably, most of the genes that showed a dissimilar alternative splicing pattern were not found among the edited transcripts. This phenomenon has been previously observed [3,4] and suggests that the regulation of splicing directly by alteration of splicing-related motifs may not be the sole mechanism by which ADAR1 mediates splicing regulation. RNA editing events within transcripts of genes related to the splicing machinery or editing-independent ADAR1 regulation may also globally affect the splicing of many transcripts.

\section{CDK13 is edited in thyroid cancer cells}

We focused on the editing event that produced the one missense mutation because of its potential impact on the resulting protein by amino acid substitution, affecting protein conformation, localization and/or interactions. This editing event occurred in the CDK13 transcript, a gene related to splicing $[19,20]$ and transcription [18] (Fig. 1B). Of note, the involvement of CDK13 in the splicing process could explain, at least in part, the effect of ADAR1 on the observed global splicing patterns (Fig. 2). The editing site was located at position 308 (c.308A > G) on the cDNA, and resulted in an amino acid change from glutamine to arginine at position 103 (Q103R) of the CDK13 N-terminal region (Fig. 3D). To confirm CDK13 editing in the Cal62 cell line used for RNA-seq, we performed Sanger sequencing for both cDNA (obtained directly from RNA) and genomic DNA (gDNA). As shown in Fig. 3E, a high frequency of CDK13 RNA editing was detected in siControl cells but not in equivalent ADAR1-silenced cells or in the gDNA sequence. Identical results were obtained in a second thyroid cancer cell line, TPC1, in which the edited form was even more abundant than the WT form in control cells (Fig. 3F). Thus, the CDK13 c.308A > G editing event is present in thyroid tumor cells and is dependent on ADAR1 expression.

Importantly, when we analyzed the WT and edited forms of CDK13 by RT-PCR and Sanger sequencing in PTC and contralateral normal tissue from 6 patients, we found a significantly higher level of edited CDK13 in the tumor tissue samples (Fig. 3G). These data suggest that $C D K 13$ editing also occurs in thyroid tissue and that, in accordance with the high A-to-I editing activity observed in thyroid cancer $[7,8]$, a higher editing rate of $C D K 13$ is evident in tumor samples. While we acknowledge that the number of thyroid tumor samples analyzed is small, these data support the in vitro observations and indicate that the editing event is a significant phenomenon.

\section{CDK13 silencing reduces proliferation, viability and invasion in thyroid cancer cells}

There are few studies on the role of CDK13 in cancer $[33,34]$ and, to our knowledge, none of them have addressed its role in thyroid cancer. To examine the function of $C D K 13$ and how its expression affects the main cancer hallmarks in thyroid cancer, we performed loss-of-function assays using three independent siRNAs that markedly decreased CDK13 RNA and protein levels in Cal62 and TPC1 thyroid cancer cells (Fig. 4A, B). We observed that CDK13 silencing inhibited cell proliferation, as measured by BrdU incorporation (Fig. 4C), and cell viability, as measured by XTT dye reduction and crystal violet staining (Fig. 4D-E). Notably, CDK13 knockdown suppressed cell invasion (Fig. 4F), a fundamental hallmark for cancer progression and dissemination, pointing to an oncogenic role for CDK13 in thyroid cancer cells.

\section{Edited CDK13-Q103R promotes aggressive phenotypes of thyroid cancer cells}

We further investigated CDK13 because of its oncogenic behavior in thyroid cancer cell lines (Fig. 4C-F) and because an edited form of its transcript is dominant in primary thyroid tumors (Fig. 3G). We hypothesized that an amino acid change in the protein could be relevant for its tumorigenic behavior in thyroid cancer cells. To test this, we established lentiviral constructs to overexpress WT-CDK13 or its edited c.308A $>$ G (Q103R) form (edit-CDK13). We transduced both Cal62 and TPC1 cells and obtained stable cell lines with constitutive overexpression of the CDK13 forms tagged with HA, or an empty vector control cell line (Supplementary Figure 2A, B). In accord with the loss-of-function assays, we observed that WT-CDK13 stimulated

\footnotetext{
(See figure on next page.)

Fig. 3 ADAR1 edits CDK13 transcript at position c.308A > G. A Distribution of 12 types of nucleotide changes across the entire transcriptome of Cal62 cells after ADAR1 knockdown, as profiled by RNA-seq. B RNA-seq analysis showing a global reduction in the total number of variants after ADAR1 silencing. C Distribution of A-to-l editing sites over annotated genomic regions. Abbreviations: UTR, untranslated region. D Details of the position and significance of the CDK13 editing event that is downregulated after ADAR1 knockdown in Cal62 cells. Fold-change and FDR refers to the allele frequency change in siControl versus SIADAR1. $\mathbf{E}$ and $\mathbf{F}$ Sanger sequencing chromatograms using a reverse primer illustrate editing of the selected CDK13 event in siControl, siADAR1 \#1, siADAR1 \#2 and genomic DNA (gDNA) in Cal62 (E) and TPC1 (F) thyroid cancer cell lines. G CDK13 c.308 A > G editing frequency in normal $(n=6)$ and PTC tumor samples $(n=6)$ calculated by taking the peak area of $\mathrm{G}$ versus A using Indigo. Error bars indicate standard deviations. Asterisks denote statistical significance as assessed with Student's t-test (two-tailed). ${ }^{*} p<0.05$
} 


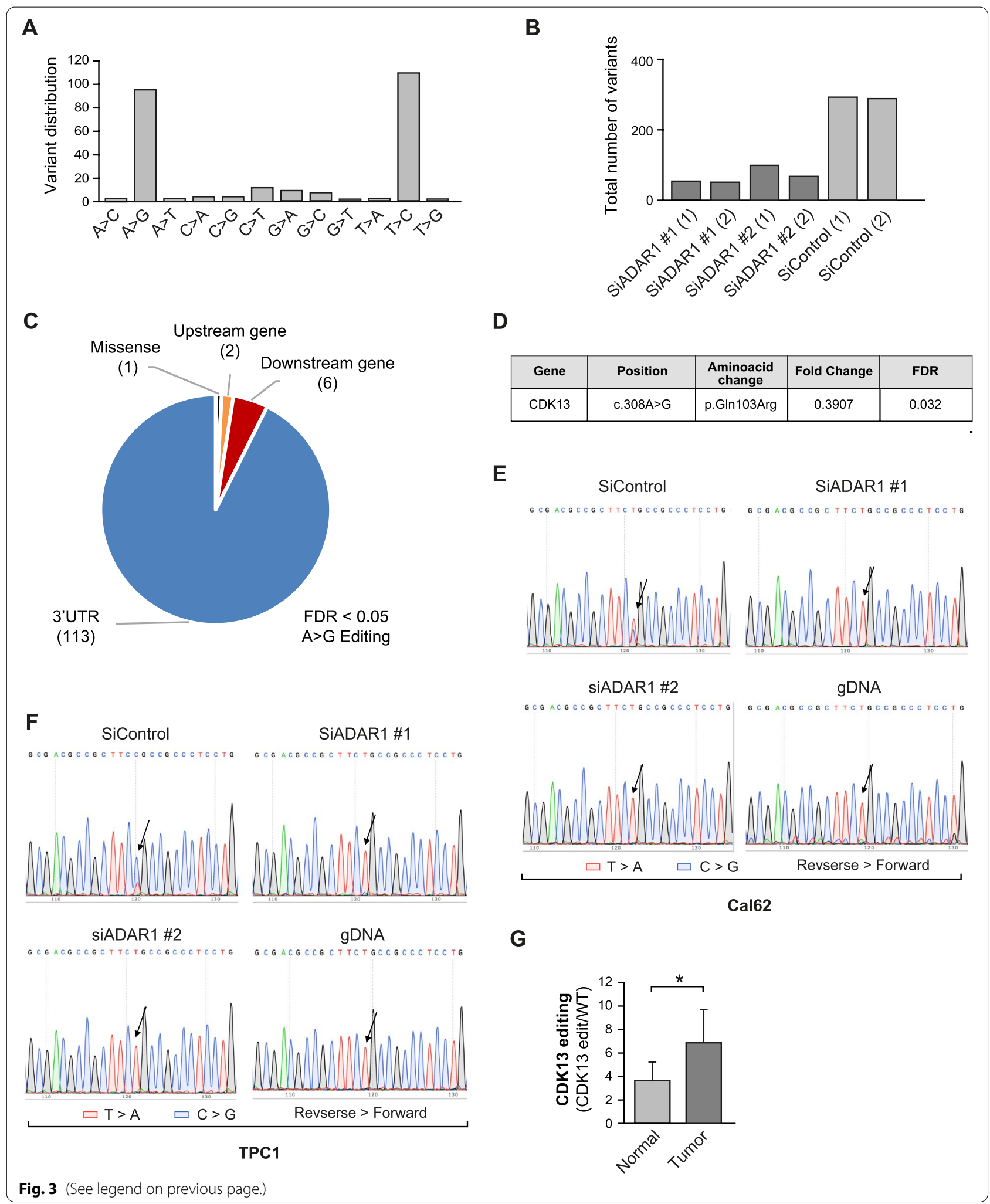


proliferation (Fig. 5A), cell viability (Fig. 5B, C) and invasion (Fig. 5D) in Cal62 and TPC1 cells. Remarkably, the aforementioned features were significantly greater in edit-CDK13 cells than in WT-CDK13 cells (Fig. 5A$D)$. These results demonstrate that the ADAR1 editing event in the CDK13 transcript potentiates the aggressive behavior of the thyroid cancer cells.

\section{Edited CDK13 rescues the proliferation and invasion defects in ADAR1-silenced cells}

To assess the importance of the CDK13 editing event in the oncogenic function of ADAR1 we overexpressed the edited form of CDK13 in thyroid cancer cells that were simultaneously silenced for ADAR1 using two different siRNAs (Supplementary Figure 3A, B). We observed that the suppressive effect of ADAR1 knockdown on proliferation (Fig. 6A), viability (Fig. 6B, C) and invasion (Fig. 6D) was rescued by the overexpression of edit-CDK13 in Cal62 and TPC1 cells. Of note, when WT-CDK13 was overexpressed as a control, we observed that in contrast to the complete rescue achieved by the edited form, the decrease in cell viability induced by $A D A R 1$ silencing was not reversed (Supplementary Figure $3 \mathrm{C}$ ). Moreover, the two cancer cell lines exhibited a different CDK13 WT/edit ratio, with a higher representation of the edited form in TPC1 cells (Fig. 3F) and higher levels of CDK13 WT in Cal62 cells (Fig. 3E). This indicates that the outcomes are independent of the endogenous CDK13 WT/edited levels, and suggests that CDK13 editing has a relevant role in the oncogenic function of $A D A R 1$ in thyroid cancer.

\section{CDK13 editing changes the nuclear localization of the protein}

CDK13 is a member of the cyclin-dependent serine/ threonine protein kinase family, with a conserved central kinase domain in the $\mathrm{C}$-terminal region and a regulatory $\mathrm{N}$-terminal region. The $\mathrm{N}$-terminal region contains arginine-serine (RS)-rich domains (Supplementary Figure $4 \mathrm{~A}$ ) that differentiate this kinase from other family members. The RS-rich domains span residues 200-435 in CDK13 [18] and link this kinase to the serine-arginine (SR) protein family, which are required for both constitutive and alternative pre-mRNA splicing. In addition, the $\mathrm{N}$-terminal region contains three bipartite nuclear localization signals (NLS) that determine the nuclear localization of the protein [20]. As the c.308A $>$ G edited site is located in position 103 of the CDK13 N-terminal region (Supplementary Figure 4A), we speculated that this amino acid change might affect the NLS sequences and, consequently, CDK13 localization. Using NLS mapper [35], we found that the amino acid change alters four bipartite NLS sequences in the N-terminal region and increases the predicted scores, indicating stronger NLS activity (Supplementary Figure 4B). We assessed CDK13 localization by protein fractionation and western blotting and observed that both WT and edited forms of CDK13 were localized to the nucleus (Supplementary Figure 4C).

Even et al. [20] have demonstrated that CDK13 can shuttle between the nucleoplasm and nuclear speckles, and that the $\mathrm{N}$-terminal region is critical for localization of the kinase in these structures. Nuclear speckles are nuclear domains enriched in pre-mRNA splicing factors, located in the interchromatin regions of the nucleoplasm of mammalian cells [36]. We used immunofluorescence to analyze the localization of the WT and edited forms of CDK13 in transduced Cal62 and TPC1 cells. We also used an antibody directed against the splicing factor SC35, which specifically marks nuclear speckles [20]. We observed that WT-CDK13 correlated highly with the nuclear speckles in thyroid cancer cells (Fig. 7A), suggesting that the described role of CDK13 in splicing $[19,20]$ is maintained in thyroid cells. By contrast, edit-CDK13 showed a significantly lower correlation with nuclear speckles in Cal62 and TPC1 cells (Fig. 7A). In addition, we observed that editCDK13 was more uniformly expressed in the nucleus, whereas WT-CDK13 was almost nonexistent in some patches, likely corresponding to the nucleolus. To validate this, we utilized an antibody against the nucleolar protein Fibrillarin, and observed that WT-CDK13 was absent from the nucleolus, whereas edit-CDK13 showed a strong co-localization with this structure (Fig. 7B). Notably, $19.6 \%$ of Cal62 and $15.2 \%$ of TPC1 cells transduced with edit-CDK13 showed enrichment of CDK13 at the nucleolus, as demonstrated by co-localization with Fibrillarin, but not at the nuclear speckles (Fig. 7C). When the same experiment was performed with WT-CDK13, no accumulation was found in the nucleolus in Cal62 cells and only $1.5 \%$ of TPC1 cells

\footnotetext{
(See figure on next page.)

Fig. 4 CDK13 is functionally important for thyroid cancer development. Cal62 and TPC1 cells were transfected with three independent siRNAs against CDK13 or a control siRNA (siControl). A CDK13 RNA relative levels $(n=3)$. B Representative western blotting for CDK13. GAPDH was used as loading control. C Proliferation by BrdU incorporation $(n=3)$. $\mathbf{D}$ and $\mathbf{E}$ Viability assays tested by XTT dye reduction $(n=3)(\mathbf{D})$ or crystal violet staining $(n=4)(\mathbf{E})$. F Matrigel Transwell invasion assay $(n=3)$. Error bars indicate standard deviations. Asterisks denote statistical significance compared with siControl treatment as assessed with Student's t-test (two-tailed). ${ }^{*} p<0.05,{ }^{* *} p<0.01,{ }^{* *} p<0.001$
} 
A

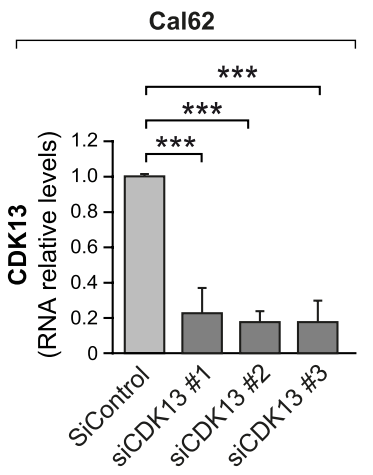

C

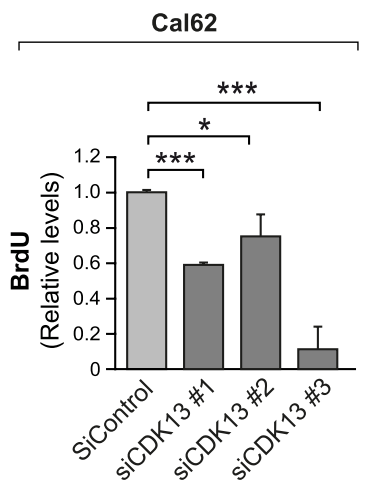

E
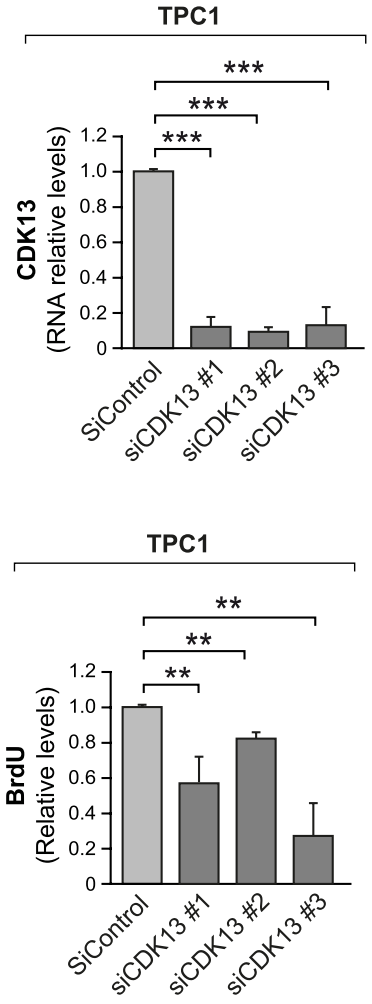

B

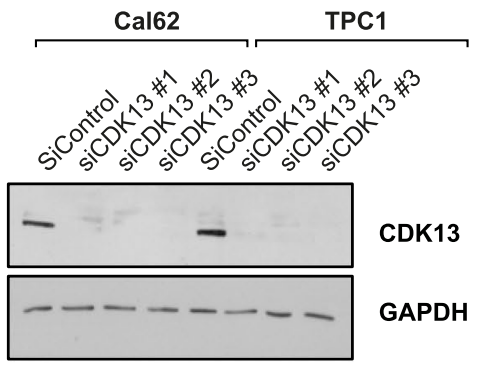

D
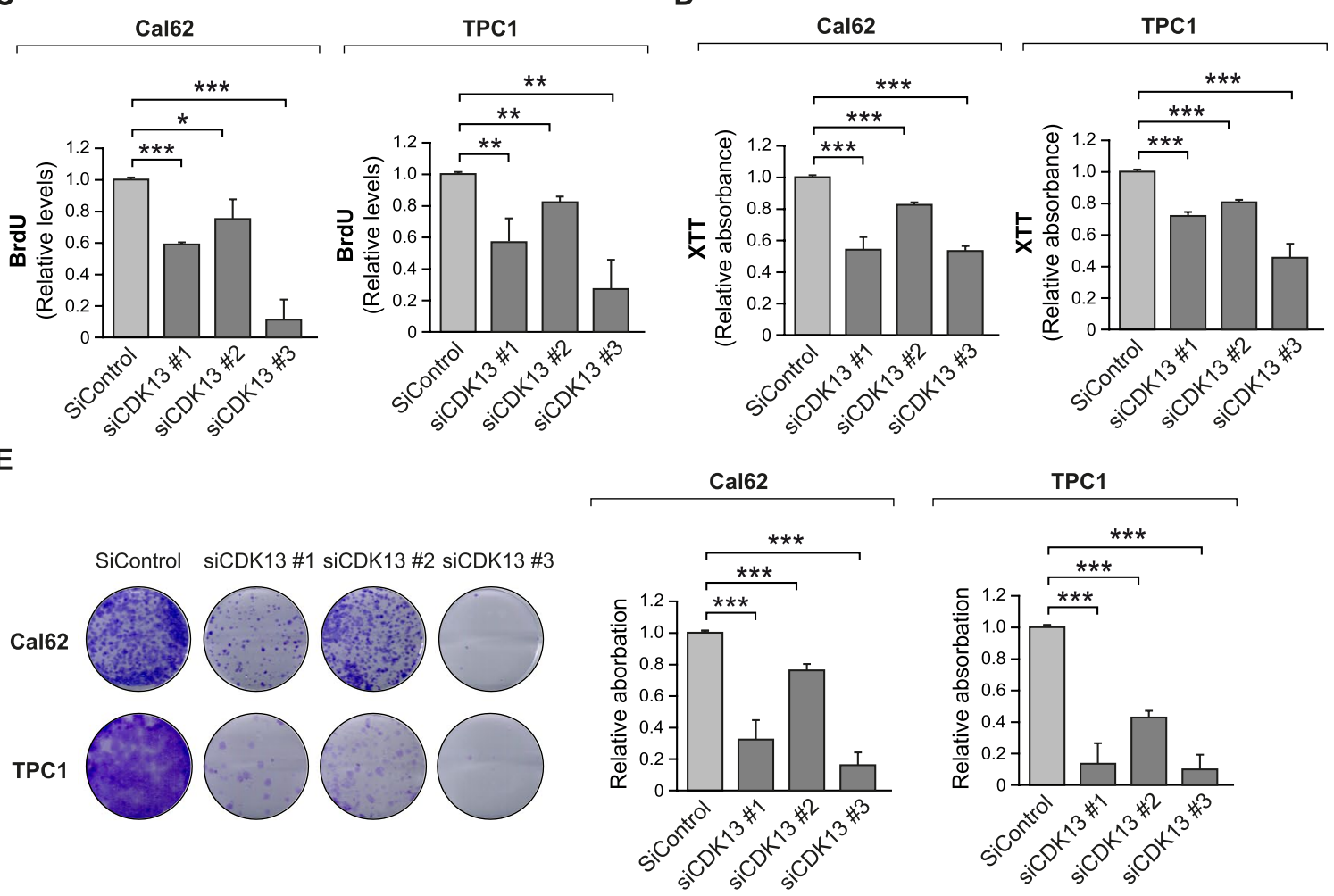

$\mathbf{F}$
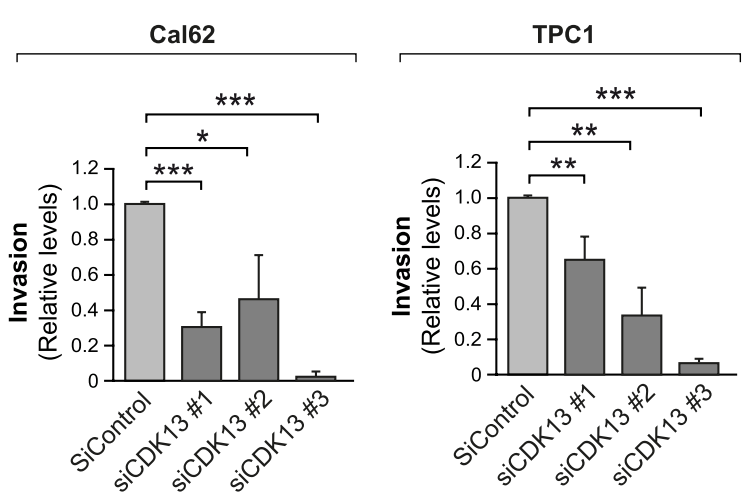

Fig. 4 (See legend on previous page.) 


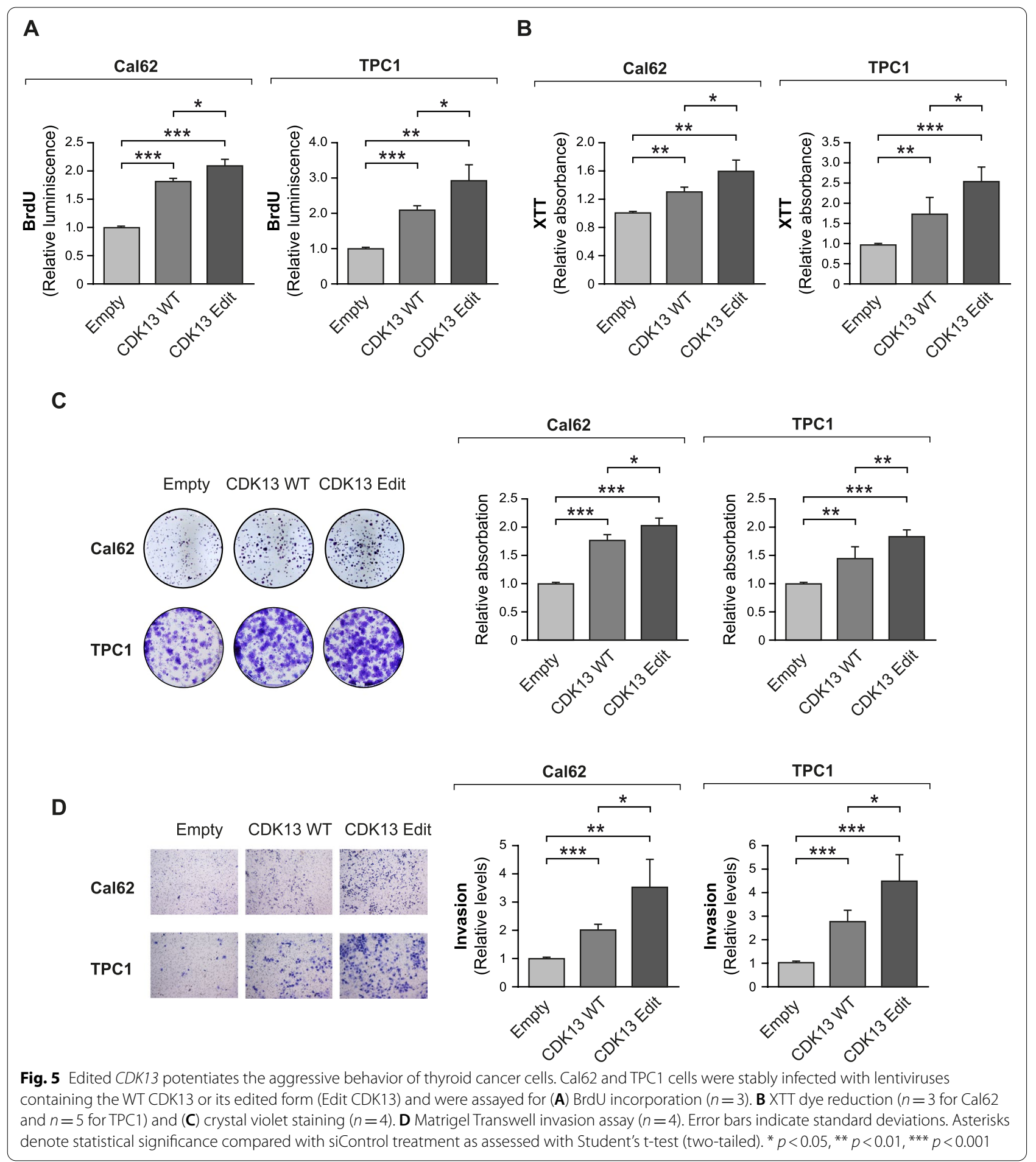

showed nucleolar accumulation. These findings demonstrate that editing of $C D K 13$ induces a change in the localization pattern of the protein, decreasing its association with nuclear speckles but enriching its localization at the nucleolus.
CDK13 editing induces changes in splicing patterns

Finally, given the described involvement of CDK13 in the splicing process $[19,20]$, we questioned whether the change in $C D K 13$ editing would contribute to some of the ADAR1-silencing-induced changes in splicing 


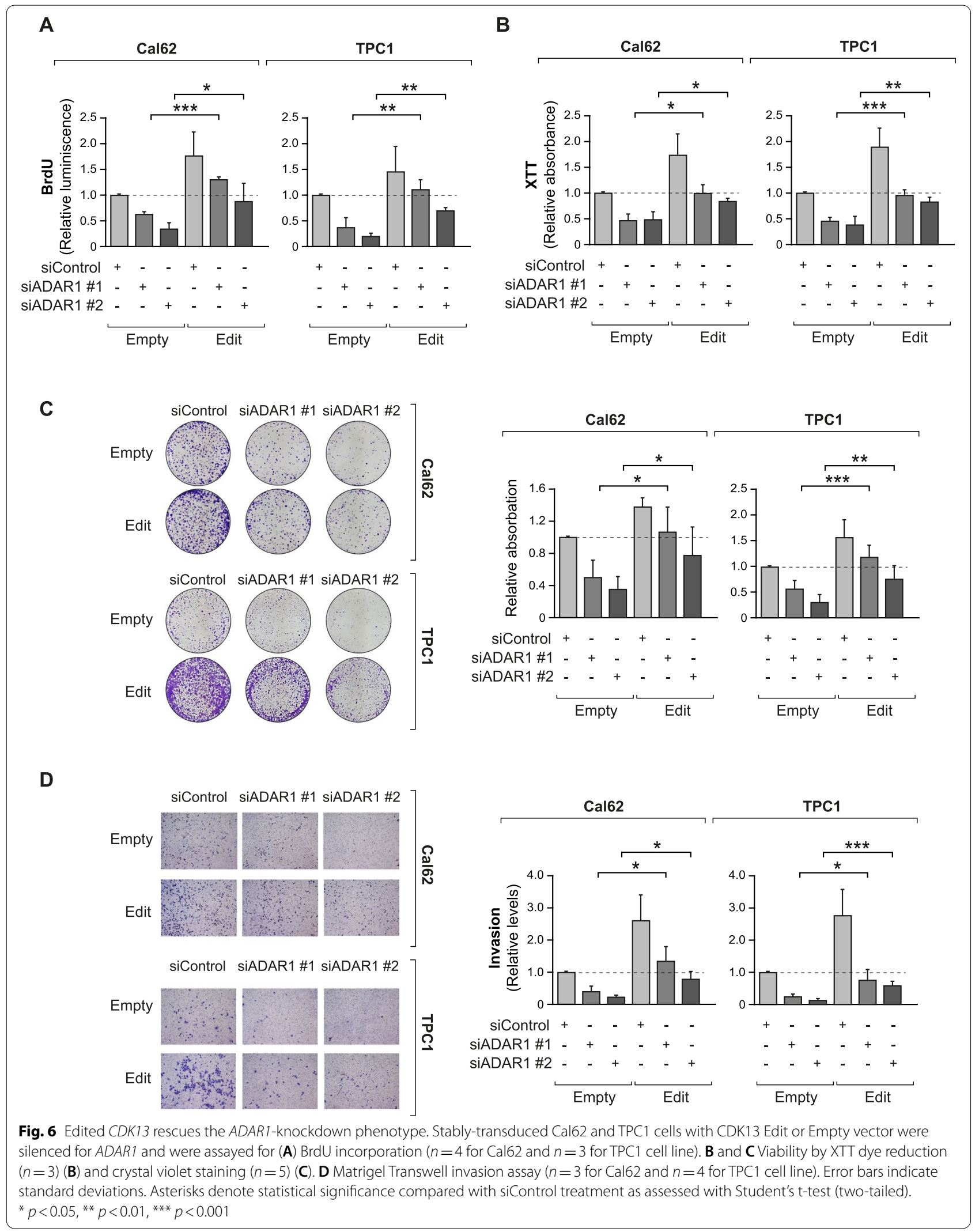


highlighted in our RNA-seq data. We analyzed the levels of the candidate splicing products altered in ADAR1silenced cells; specifically, those potentially involved in the cytoskeleton and later validated by qPCR. We observed that some splicing products were increased (A5SS event involving the exon 57,761,797-57,762,046 in CTNND1, SE of exon 2,241,520-2,241,790 of HAUS3, and the MXE splicing event involving the exon $35,685,064-35,685,139$ in TMP2) or decreased (SE 155,061,904-155,062,117 in ADAM15) following transfection of edit-CDK13 in both Cal62 and TPC1 cells (Fig. 8). Some splicing events were not consistently altered in both cell lines such as the SE events in NUMA1, SGO1 and LRCH2 in TPC1 cells, or the SE in TMP2 and ENAH in Cal62 cells (Fig. 8). As expected, the splice products increased in edit-CDK13 cells were lower in ADAR1-silenced cells, where the edited form of CDK13 is almost absent, and vice versa. Interestingly, we observed a different pattern of splicing events in cells overexpressing WT and edited CDK13, with the transduction of the edited form responsible for most of the alternative splicing changes.

\section{Discussion}

Post-transcriptional modification of RNA is a widespread phenomenon that expands the transcriptome and the range of functions of RNA transcripts. One of the most surprising discoveries in this field is that mammalian RNAs can undergo RNA editing, which modifies specific RNA nucleotides without altering the DNA template $[37,38]$. A-to-I editing can profoundly influence cellular functions by altering mRNA splicing, stability, localization, and translation, and by interfering with the binding of regulatory RNAs [39]. The magnitude and the biological consequences of A-to-I editing in the majority of cancers remain largely unknown.

Advances in high-throughput sequencing and data generation have revealed that RNA editing events are extensive across the human cancer transcriptome, and that the incidence and progression of multiple cancers are associated with some of these events $[7,8,39-41]$. In the present study, we aimed to assess the impact of RNA editing in thyroid cancer and to identify de novo cancer-related
RNA editing sites using next generation sequencing in an ADAR1-knockdown cellular model. We believe that thyroid cancer represents a suitable model for this study as RNA editing activity is enriched in thyroid tumors over normal tissue $[7,8]$ and because we previously demonstrated the profound functional consequences of $A D A R 1$ knockdown for tumor progression [10]. To the best of our knowledge, no other study has systematically studied RNA editing in thyroid cancer. Our transcriptome analysis revealed novel changes triggered by ADAR1 deregulation in almost one thousand transcripts, most of them involved in fundamental processes for cancer development and progression such as cell cycle, DNA replication, regulation of the cytoskeleton and apoptosis. This finding is consistent with our previous results that functionally associated ADAR1 expression with proliferation, invasion, migration and in vivo tumor growth [10]. Moreover, $A D A R 1$ silencing induced the downregulation of several oncogenes and the upregulation of tumor suppressor genes [10], confirming the oncogenic role of $A D A R 1$ in thyroid cancer observed in other cancer types [42-47]. Beyond its oncogenic activity, ADAR1 has been shown to elicit an important role in immunity [48, 49], and mutations or alterations in ADAR1 can confer autoimmunity in humans and in animal models [49-54]. Along this line, our RNA-seq results reveal that the innate immune response (GO: 0,045,087) and cytokine production (GO: 0,001,817) are biological processes enriched upon ADAR1 silencing (data not show). Because autoimmune thyroid disease is closely related to thyroid cancer [55], it is becoming increasingly important to identify potential diagnostic biomarkers and therapeutic targets for both diseases and ADAR1 could be a good candidate. Future studies should be orientated in this direction.

The deregulation of genes involved in immunity is likely elicited by the silencing of the p150 ADAR1 isoform. However, other biological processes related to the p110 isoform [56], also silenced in our study, were deregulated, such as DNA damage checkpoint (GO:0,000,077). It is highly probable that both ADAR1 isoforms contribute to the growth defect observed in vitro and in vivo after ADAR1 silencing [57]; however, our study design did not allow us to distinguish the specific isoform responsible

\footnotetext{
(See figure on next page.)

Fig. 7 Edited and wild-type CDK13 show differences in nuclear speckle and nucleolus localization. Stably-transduced CDK13-WT, CDK13-Edit or Empty vector Cal62 and TPC1 cell lines were used. $\mathbf{A}$ and $\mathbf{B}$ Confocal images of immunofluorescence staining of the cells with antibodies against HA (green), the nuclear speckle marker SC35 (red) (A) or the nucleolus marker Fibrillarin (Pink) (B). Nuclei were stained with DAPI (blue). Colocalization was analyzed using Fiji-Coloc2 plugin, and the mean of the Pearson R value from 3 independent experiments is represented in the bottom panels. C Upper panels: Representative image of CDK13-Edit Cal62 and TPC1 cells with nucleolar accumulation of CDK13. The images represent the immunofluorescence staining of cells with antibodies against HA (green), SC35 (red) or Fibrillarin (pink). Bottom panel: Graphs show quantification of the percentage of CDK13-WT or CDK13-Edit cells that present with nucleolar accumulation of CDK13 ( $n=3$ independent experiments). Error bars indicate standard deviations. Asterisks denote statistical significance as assessed with Student's t-test (two-tailed). ${ }^{*} p<0.05,{ }^{* *} p<0.01,{ }^{* * *} p<0.001$
} 


$$
\text { A }
$$

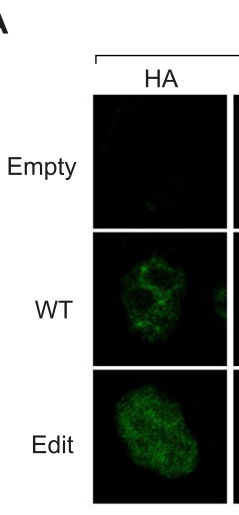

\section{Cal62} SC35/HA SC35/HA/DAP
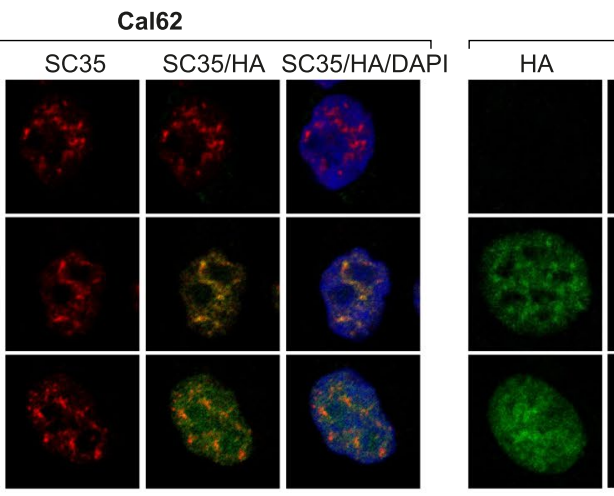

TPC1

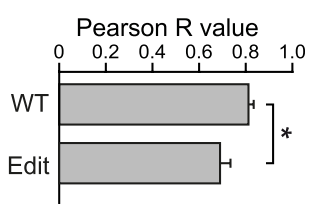

B

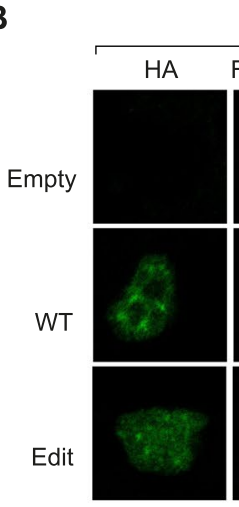

Cal62

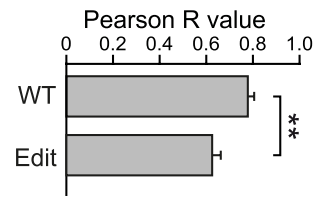

Cal62
FIBRILLARIN FIBR/HA FIBR/HA/DAPI
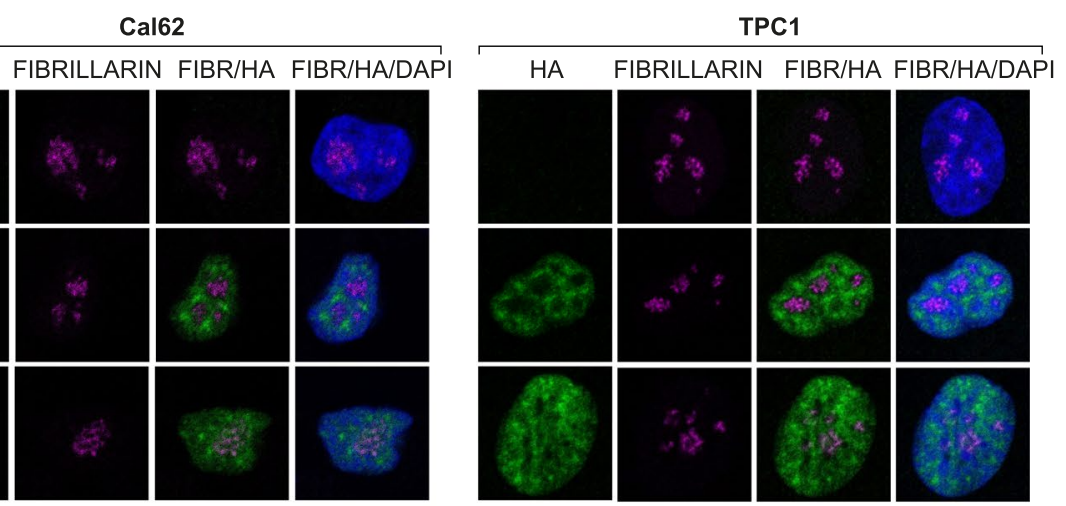

Pearson $\mathrm{R}$ value
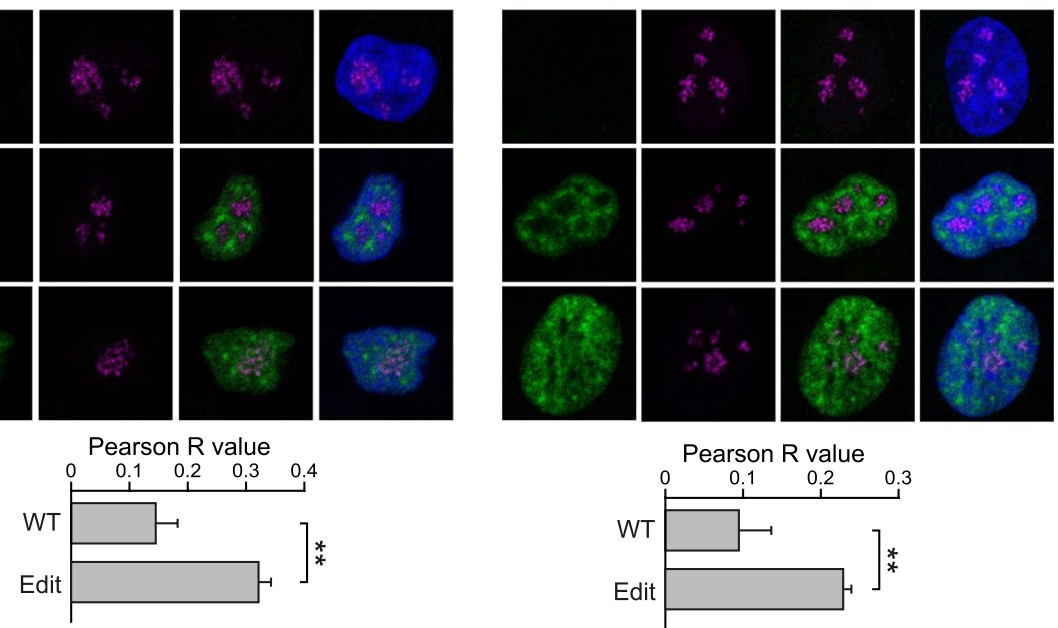

C

Cal62

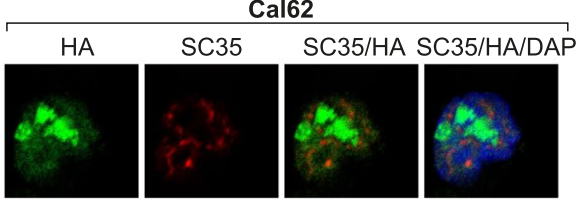

TPC1
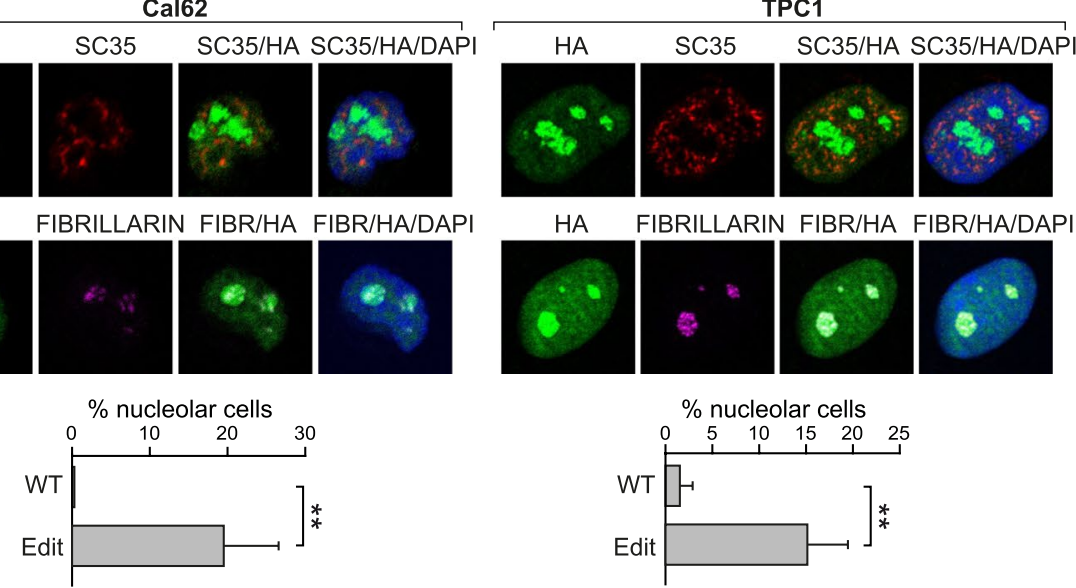

Fig. 7 (See legend on previous page.) 


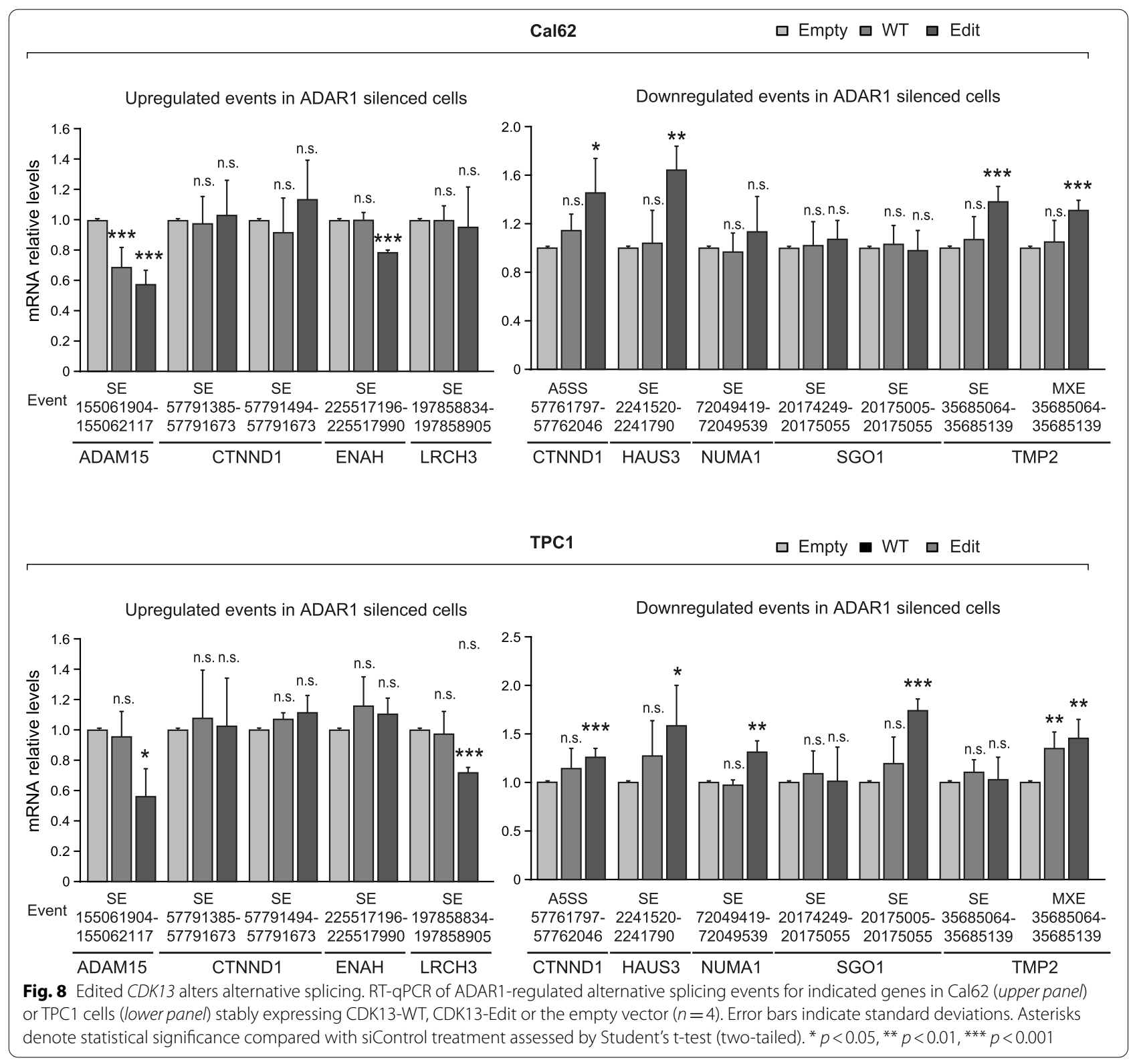

for CDK13 editing and this should be addressed in a future study.

Interestingly, alternative splicing was enriched among the deregulated genes after $A D A R 1$ silencing. Detailed analysis revealed that $A D A R 1$ has a prominent influence on the global splicing pattern and identified approximately one hundred high-confidence splicing events affected by ADAR1. These gene sets are enriched with similar functions, for example, we observed several genes involved in the cytoskeleton and thus, likely contributing to the altered invasion, migration or 3D growth previously observed upon ADAR1 loss-of-function [10]. Other studies have also provided evidence for crosstalk between RNA editing enzymes and the splicing machinery $[3,4,58]$. Nevertheless, detailed mechanistic explanations and their biological importance in cancer are lacking. Regulation of alternative splicing involves cisacting elements within the pre-mRNAs, and trans-acting factors such as SR proteins and heterogeneous nuclear ribonucleoproteins [3, 59-63]. RNA-seq analysis demonstrated that most of the transcripts with significantly altered splicing did not present with changes in edited adenosines, suggesting that the ADAR1-dependent regulation of alternative splicing is not only dependent on the direct editing of cis-acting elements. Other studies have obtained comparable results in other cell types, 
with few alternately spliced sites explained by nearby A-to-I edit sites [3, 4]. Our identification of an editing event in $C D K 13$, which encodes for an SR-related protein implicated in splicing $[19,20]$, might shed some light on this phenomenon. We propose that the extent of ADAR1-regulated splicing is mediated predominantly by trans-acting elements like CDK13, rather than direct ciselement editing, leading to a broad influence on splicing for many genes. Indeed, our results demonstrate that CDK13 editing is responsible for some of the splicing events identified by our RNA-seq analysis. Interestingly, genes showing significant changes in their splicing pattern are frequently involved in the splicing activity itself. This has been also been observed in hepatocellular carcinoma, myelogenous leukemia and glioblastoma [3]. This may suggest that a first editing event that influences the splicing process, such as CDK13 editing, could produce splicing changes of other alternative splicing regulators, ultimately having a prominent influence on the global splicing pattern observed.

We believe that our results obtained in thyroid cancer cells and tumor samples can be extrapolated to other cancer types. ADAR1 and CDK13 have been demonstrated to act as oncogenes in several cancer types [33, 34, 42, 64-66]. Moreover, in agreement with the oncogenic behavior conferred by CDK13 editing described herein, the $C D K 13$ editing event $(\mathrm{c} .308 \mathrm{~A}>\mathrm{G})$ has been found to be overrepresented in several tumor types as compared with normal control samples, such as hepatocellular carcinoma [67], glioblastoma [68] and kidney renal clear cell carcinoma [7]. Supporting our results in thyroid cancer, CDK13 editing correlated with poor prognosis in hepatocellular carcinoma [67]. Moreover, we observed that this editing event is present in the triple-negative breast cancer cell lines BT20 and MB468, and is also dependent on ADAR1 expression (our unpublished observations). We believe that this is particularly relevant as ADAR1 and CDK13 have both been described as important factors and possible therapeutic targets in triple-negative breast cancer $[33,69]$.

To our knowledge, this is the first study to analyze the functional importance of CDK13 editing in cancer. Few studies had previously demonstrated the function of CDK13 in tumorigenesis. CDK13 was described as amplified in hepatocellular carcinoma and exhibits oncogenic activity [34]. Other studies showed that the simultaneous inhibition of CDK12 and CDK13 suppresses tumorigenic features in leukemia [64], ovarian cancer [65] and triple-negative breast cancer [33]. However, Quereda et al. [33] were the only group to demonstrate that downregulation of CDK13 (without simultaneous CDK12 inhibition) inhibited colony formation in a breast cancer cell line. Our functional analysis shows that, in accordance with previous studies [33, 34, 64, 65], CDK13 acts an oncogene in thyroid cancer cells, identifying it as a new therapeutic target in thyroid and other cancer types. The fact that TCGA data show no CDK13 upregulation in PTC would indicate that the mechanism of CDK13 oncogenic activation in PTC is via editing rather than upregulation. As we observed, edited Q103R CDK13 stimulates a stronger cell proliferation and migration phenotype than WT CDK13 and, probably because of this, CDK13 editing might be an efficient mechanism contributing to tumor progression and aggressiveness.

In summary, our work confirms $A D A R 1$ as an oncogene in thyroid cancer and offers evidence of its mechanism of action through editing $C D K 13$, which confers stronger tumorigenic properties to cells and delocalizes CDK13 from nuclear speckles, the main hub for splicing factors. Moreover, editing of this SR-related protein changes the splicing of several transcripts and may explain, at least in part, the splicing pattern induced by ADAR1 deregulation. This work opens the door for detailed studies of the mechanism of action of CDK13 in thyroid cancer with respect to how edited CDK13 triggers the observed oncogenic behavior. In this line, we observed a change in the splicing pattern of some potentially interesting candidate genes. For example, ADAM15 splicing is altered after edit-CDK13 overexpression in both Cal62 and TPC1 cell lines. This is of special relevance because aberrant $A D A M 15$ expression has been associated with human cancer [70] and the alternative splicing of ADAM15 is mis-regulated in cancer cells [71]. Indeed, the splicing form upregulated in edit-CDK13 cells has been associated with poor prognosis in patients with node-negative breast cancer [72]. Other interesting candidates previously related to cancer include CTNND1 [73] and TPM2 [74, 75], for which alternative splicing events in both transcripts have been correlated with cancer [76, 77], and HAUS3 [78].

Finally, our work supports ADAR1-mediated A-to-I editing as an important pathway in cancer progression, and highlights $A D A R 1, C D K 13$ and the edited CDK13Q103R as potential targets for the development of new treatments for thyroid and other cancer types.

\footnotetext{
Abbreviations

A3SS: 3'Splice sites; A5SS: 5'Splice sites; ADAR1: Adenosine deaminases acting on RNA; ATC: Anaplastic Thyroid Carcinoma; BrdU: Bromodeoxyuridine; CDK13: Cyclin-Dependent Kinase 13; FDR: False discovery rate; FTC: Follicular Thyroid Carcinoma; GAPDH: Glyceraldehyde Phosphate Dehydrogenase; GEO: Gene Expression Omnibus; MATS: Multivariate Analysis of Transcript Splicing; MXE: Mutually exclusive exons; NLS: Nuclear Localization Signals; PBS: PhosphateBuffered Saline; PDTC: Poorly Differentiated Thyroid Carcinoma; PTC: Papillary Thyroid Carcinoma; qRT-PCR: Quantitative real-time PCR; RI: Retained introns; RNA-seq: RNA-sequencing; SDS-PAGE: Sodium Dodecyl Sulfate-Polyacrylamide Gel Electrophoresis; SE: Skipped exons; SNP: Single Nucleotide
} 
Polymorphism; TCGA: The Cancer Genome Atlas; TPM: Transcript Per million; UTR: Untranslated region; WT: Wild-type.

\section{Supplementary Information}

The online version contains supplementary material available at https://doi. org/10.1186/s12943-021-01401-y.

\section{Additional file 1: Table S1. Primers}

Additional file 2: Supplementary Figure 1. ADAR1 silencing. Representative western blot of ADAR1 steady-state expression at the indicated time points after ADAR1 silencing in Cal62 and TPC1 cell lines. GAPDH was used as a loading control.

Additional file 3: Supplementary Figure 2. CDK13 levels in cells stably expressing CDK13-WT, CDK13-Edit or the Empty vector. Cal62 and TPC1 were infected with lentivirus expressing the WT or the edited form of CDK13 (CDK13-WT and CDK13-Edit, respectively) or the corresponding empty vector, and selected using puromycin. (A) CDK13 levels in the indicated cell lines. (B) Representative western blotting for HA antibody in the indicated cell lines. Vinculin was used as loading control $(n=3)$. Error bars indicate standard deviations. Asterisks denote statistical significance compared with siControl treatment assessed by Student's t-test (twotailed). n.s: non significative $p>0.05,{ }^{* *} p<0.01,{ }^{* * *} p<0.001$.

Additional file 4: Supplementary Figure 3. CDK13 overexpression and ADAR1 silencing in Cal62 and TPC1 cell lines. RNA relative levels for CDK13 (A) and ADAR1 (B) in Cal62 and TPC1 cell lines stably expressing CDK13-Edit 72 hours after ADAR1 siRNA (siADAR1 \#1 and \#2) or Control siRNA (siControl) transfection. (C) Stably-transduced Cal62 and TPC1 cells with CDK13-Edit, CDK13-WT or empty vector were silenced for ADAR1 and were assayed for cell viability by XTT dye reduction $(n=4)$. Asterisks denote statistical significance assessed by Student's t-test (two-tailed). n.s: non significative $p>0.05,{ }^{* *} p<0.01,{ }^{* * *} p<0.001$.

Additional file 5: Supplementary Figure 4. Both CDK13-WT and CDK13Edit are located in the cell nucleus. (A) Schematic representation of the CDK13 domain structure and the CDK13 editing event. Numbers below indicate the amino acid position. Abbreviations: RS: arginine/serine-rich; KD: kinase domain; C: C-terminal extension. (B) Predicted bipartite NLS (Nuclear Localization Sequence) in the WT and c.308 A>G edited form of CDK13. Predictions were performed using NLS mapper (http://nls-mapper. iab.keio.ac.jp). (C) Representative western blotting for HA and CDK13 in the indicated Cal62 and TPC1 cells after cytoplasm-nucleus fractionation. SP1 and tubulin were used as loading control for the nucleus and the cytoplasm, respectively.

\section{Acknowledgements}

We thank the Harvard Bioinformatics Core for the RNA sequencing analysis. We also thank Dr. Garcilaso Riesco-Eizaguirre, Hospital Universitario de Mostoles (Madrid), for providing thyroid tumor samples and clinical information of the patients, and Dr. K McCreath for helpful comments on the manuscript.

\section{Authors' contributions}

JR-M and ARB designed the experiments and JR-M conducted the experiments, acquired and analyzed the data. CM performed the splicing bioinformatic analysis. J.R-M and PS wrote the manuscript and AR-B, CM, RI-G and FJ-S provided conceptual ideas and reviewed the manuscript. AR-B, PS and FJ-S contributed to critical discussion. The author(s) read and approved the final manuscript.

\section{Funding}

This work was supported by the following grants: SAF2016-75531-R, Fondo Europeo de Desarrollo Regional, and PID2019-105303RB-100/ AEI/10.13039/501100011033 from Ministerio de Ciencia e Innovación, Spain; B2017/BMD-3724 from Comunidad de Madrid, and GCB14142311CRES from Fundación Española Contra el Cáncer (AECC) (to PS); NIH (R35 CA232105) and the Ludwig Center at Harvard (to FJ-S); outstanding Investigator Award (R35CA232115) from the National Cancer Institute of the NIH (to RI-G). J.R-M holds an FPU fellowship from Ministerio de Educación, Cultura y Deporte (Spain).

\section{Declarations}

Ethics approval and consent to participate

Ethics approval and consent to participate was obtained from all the patients following the protocols approved by the hospital ethics committee (Hospital Universitario La Paz, Madrid, Spain).

\section{Consent for publication}

The patients involved in our study gave consent for publication to the Biobank of the Hospital.

\section{Competing interests}

$\mathrm{RI}-\mathrm{G}$ and FJ-S are co-founders and scientific advisory board members of 28-7 Therapeutics and Theon Therapeutics.

\section{Author details}

${ }^{1}$ Instituto, de Investigaciones Biomédicas "Alberto Sols"; Consejo Superior de Investigaciones Científicas (CSIC), Universidad Autónoma de Madrid (UAM), Madrid, Spain. ${ }^{2}$ Department of Pathology, Harvard Medical School Initiative for RNA Medicine, Beth Israel Deaconess Medical Center, Harvard Medical School, Boston, MA, USA. ${ }^{3}$ Stem Cell Program, Division of Hematology/ Oncology, Boston Children's Hospital, Departments of Biological Chemistry and Molecular Pharmacology, and Pediatrics, Harvard Medical School, Harvard Medical School Initiative for RNA Medicine, Boston, MA, USA. ${ }^{4}$ Centro de Investigación Biomédica en Red de Cáncer (CIBERONC) Instituto de Salud Carlos III (ISCIII), Madrid, Spain.

Received: 4 March 2021 Accepted: 3 August 2021

Published online: 08 September 2021

\section{References}

1. Bass BL. RNA editing by adenosine deaminases that act on RNA. Annu Rev Biochem. 2002;71:817-46.

2. Nishikura K. A-to-l editing of coding and non-coding RNAs by ADARs. Nat Rev Mol Cell Biol. 2016;17:83-96.

3. Solomon O, Oren S, Safran M, Deshet-Unger N, Akiva P, Jacob-Hirsch J, Cesarkas K, Kabesa R, Amariglio N, Unger R, et al. Global regulation of alternative splicing by adenosine deaminase acting on RNA (ADAR). RNA. 2013;19:591-604.

4. Tang SJ, Shen H, An O, Hong H, Li J, Song Y, Han J, Tay DJT, Ng VHE, Bellido Molias F, et al. Cis- and trans-regulations of pre-mRNA splicing by RNA editing enzymes influence cancer development. Nat Commun. 2020;11:799.

5. Wang C, Zou J, Ma X, Wang E, Peng G. Mechanisms and implications of ADAR-mediated RNA editing in cancer. Cancer Lett. 2017;411:27-34.

6. Fritzell K, Xu LD, Lagergren J, Öhman M. ADARs and editing: the role of A-to-I RNA modification in cancer progression. Semin Cell Dev Biol. 2018;79:123-30.

7. Han L, Diao L, Yu S, Xu X, Li J, Zhang R, Yang Y, Werner HMJ, Eterovic AK, Yuan Y, et al. The genomic landscape and clinical relevance of A-to-I RNA editing in human cancers. Cancer Cell. 2015;28:515-28.

8. Paz-Yaacov N, Bazak L, Buchumenski I, Porath HT, Danan-Gotthold M, Knisbacher BA, Eisenberg E, Levanon EY. Elevated RNA editing activity is a major contributor to transcriptomic diversity in tumors. Cell Rep. 2015;13:267-76.

9. Wang Y, Xu X, Yu S, Jeong KJ, Zhou Z, Han L, Tsang YH, Li J, Chen H, Mangala LS, et al. Systematic characterization of A-to-I RNA editing hotspots in microRNAs across human cancers. Genome Res. 2017;27:1112-25.

10. Ramírez-Moya J, Baker AR, Slack FJ, Santisteban P. ADAR1-mediated RNA editing is a novel oncogenic process in thyroid cancer and regulates miR200 activity. Oncogene. 2020;39:3738-53.

11. Ferlay J, Shin HR, Bray F, Forman D, Mathers C, Parkin DM. Estimates of worldwide burden of cancer in 2008: GLOBOCAN 2008. Int J Cancer. 2010;127:2893-917.

12. Carlberg M, Hedendahl L, Ahonen M, Koppel T, Hardell L. Increasing incidence of thyroid cancer in the Nordic countries with main focus on Swedish data. BMC Cancer. 2016;16:426.

13. Safavi A, Azizi F, Jafari R, Chaibakhsh S, Safavi AA. Thyroid cancer epidemiology in Iran: a time trend study. Asian Pac J Cancer Prev. 2016;17:407-12. 
14. Lim H, Devesa SS, Sosa JA, Check D, Kitahara CM. Trends in thyroid cancer incidence and mortality in the United States, 1974-2013. JAMA. 2017:317:1338-48.

15. Xing M. Molecular pathogenesis and mechanisms of thyroid cancer. Nat Rev Cancer. 2013;13:184-99.

16. Nikiforov YE, Nikiforova MN. Molecular genetics and diagnosis of thyroid cancer. Nat Rev Endocrinol. 2011;7:569-80.

17. Nikiforova MN, Nikiforov YE. Molecular genetics of thyroid cancer: implications for diagnosis, treatment and prognosis. Expert Rev Mol Diagn. 2008;8:83-95.

18. Greifenberg AK, Hönig D, Pilarova K, Düster R, Bartholomeeusen K, Bösken CA, Anand K, Blazek D, Geyer M. Structural and functional analysis of the Cdk13/Cyclin K complex. Cell Rep. 2016;14:320-31.

19. Chen HH, Wong YH, Geneviere AM, Fann MJ. CDK13/CDC2L5 interacts with L-type cyclins and regulates alternative splicing. Biochem Biophys Res Commun. 2007;354:735-40.

20. Even Y, Durieux S, Escande ML, Lozano JC, Peaucellier G, Weil D, Genevière AM. CDC2L5, a Cdk-like kinase with RS domain, interacts with the ASF/ SF2-associated protein p32 and affects splicing in vivo. J Cell Biochem. 2006;99:890-904.

21. Edgar R, Domrachev M, Lash AE. Gene expression omnibus: NCBI gene expression and hybridization array data repository. Nucleic Acids Res. 2002;30:207-10.

22. Didion JP, Martin M, Collins FS. Atropos: specific, sensitive, and speedy trimming of sequencing reads. PeerJ. 2017;5:e3720.

23. Liao Y, Smyth GK, Shi W. featureCounts: an efficient general purpose program for assigning sequence reads to genomic features. Bioinformatics. 2014;30:923-30.

24. Patro R, Duggal G, Love MI, Irizarry RA, Kingsford C. Salmon provides fast and bias-aware quantification of transcript expression. Nat Methods. 2017;14:417-9.

25. Love MI, Huber W, Anders S. Moderated estimation of fold change and dispersion for RNA-seq data with DESeq2. Genome Biol. 2014;15:550.

26. Soneson C, Love MI, Robinson MD. Differential analyses for RNA-seq: transcript-level estimates improve gene-level inferences. F1000Res. 2015;4:1521.

27. McKenna A, Hanna M, Banks E, Sivachenko A, Cibulskis K, Kernytsky A, Garimella K, Altshuler D, Gabriel S, Daly M, DePristo MA. The genome analysis toolkit: a MapReduce framework for analyzing next-generation DNA sequencing data. Genome Res. 2010;20:1297-303.

28. Cingolani P, Platts A, Wang IL, Coon M, Nguyen T, Wang L, Land SJ, Lu X, Ruden DM. A program for annotating and predicting the effects of single nucleotide polymorphisms, SnpEff: SNPs in the genome of Drosophila melanogaster strain W1118; iso-2; iso-3. Fly. 2012;6:80-92.

29. Ramírez-Moya J, Wert-Lamas L, Santisteban P. MicroRNA-146b promotes PI3K/AKT pathway hyperactivation and thyroid cancer progression by targeting PTEN. Oncogene. 2018;37:3369-83.

30. Ramírez-Moya J, Wert-Lamas L, Riesco-Eizaguirre G, Santisteban P. Impaired microRNA processing by DICER1 downregulation endows thyroid cancer with increased aggressiveness. Oncogene. 2019;38:5486-99.

31. Ast G. How did alternative splicing evolve? Nat Rev Genet. 2004;5:773-82.

32. Shen S, Park JW, Lu ZX, Lin L, Henry MD, Wu YN, Zhou Q, Xing Y. rMATS: robust and flexible detection of differential alternative splicing from replicate RNA-Seq data. Proc Natl Acad Sci U S A. 2014;111:E5593-5601.

33. Quereda V, Bayle S, Vena F, Frydman SM, Monastyrskyi A, Roush WR, Duckett DR. Therapeutic targeting of CDK12/CDK13 in triple-negative breast cancer. Cancer Cell. 2019;36:545-558.e547.

34. Kim HE, Kim DG, Lee KJ, Son JG, Song MY, Park YM, Kim JJ, Cho SW, Chi SG, Cheong HS, et al. Frequent amplification of CENPF, GMNN and CDK13 genes in hepatocellular carcinomas. PLoS One. 2012;7:e43223.

35. Kosugi S, Hasebe M, Tomita M, Yanagawa H. Systematic identification of cell cycle-dependent yeast nucleocytoplasmic shuttling proteins by prediction of composite motifs. Proc Natl Acad Sci U S A. 2009;106:10171-6.

36 Spector DL, Lamond Al. Nuclear speckles. Cold Spring Harb Perspect Biol. 2011;3:a000646.

37. Blow MJ, Grocock RJ, van Dongen S, Enright AJ, Dicks E, Futreal PA, Wooster R, Stratton MR. RNA editing of human microRNAs. Genome Biol. 2006;7:R27.

38. Kawahara Y, Zinshteyn B, Sethupathy P, lizasa H, Hatzigeorgiou AG, Nishikura K. Redirection of silencing targets by adenosine-to-inosine editing of miRNAs. Science. 2007;315:1137-40.
39. Fumagalli D, Gacquer D, Rothé F, Lefort A, Libert F, Brown D, Kheddoumi N, Shlien A, Konopka T, Salgado R, et al. Principles governing A-to-I RNA editing in the breast cancer transcriptome. Cell Rep. 2015;13:277-89.

40. Han SW, Kim HP, Shin JY, Jeong EG, Lee WC, Kim KY, Park SY, Lee DW, Won JK, Jeong SY, et al. RNA editing in RHOQ promotes invasion potential in colorectal cancer. J Exp Med. 2014;211:613-21.

41. Chen J, Wang L, Wang F, Liu J, Bai Z. Genomic Identification of RNA editing through integrating omics datasets and the clinical relevance in hepatocellular carcinoma. Front Oncol. 2020;10:37.

42. Gannon HS, Zou T, Kiessling MK, Gao GF, Cai D, Choi PS, Ivan AP, Buchumenski I, Berger AC, Goldstein JT, et al. Identification of ADAR1 adenosine deaminase dependency in a subset of cancer cells. Nat Commun. 2018;9:5450.

43. Jiang $Q$, Crews LA, Barrett CL, Chun HJ, Court AC, Isquith JM, Zipeto MA, Goff DJ, Minden M, Sadarangani A, et al. ADAR1 promotes malignant progenitor reprogramming in chronic myeloid leukemia. Proc Natl Acad Sci U S A. 2013;110:1041-6.

44. Liu X, Fu Y, Huang J, Wu M, Zhang Z, Xu R, Zhang P, Zhao S, Liu L, Jiang H. ADAR1 promotes the epithelial-to-mesenchymal transition and stem-like cell phenotype of oral cancer by facilitating oncogenic microRNA maturation. J Exp Clin Cancer Res. 2019;38:315.

45. Qi L, Chan TH, Tenen DG, Chen L. RNA editome imbalance in hepatocellular carcinoma. Cancer Res. 2014;74:1301-6.

46. Qin YR, Qiao JJ, Chan TH, Zhu YH, Li FF, Liu H, Fei J, Li Y, Guan XY, Chen L. Adenosine-to-inosine RNA editing mediated by ADARs in esophageal squamous cell carcinoma. Cancer Res. 2014;74:840-51.

47. Wang H, Hou Z, Wu Y, Ma X, Luo X. p150 ADAR1 isoform involved in maintenance of HeLa cell proliferation. BMC Cancer. 2006;6:282.

48. Lamers MM, van den Hoogen BG, Haagmans BL. ADAR1: "editor-in-chief" of cytoplasmic innate immunity. Front Immunol. 2019;10:1763.

49. Samuel CE. Adenosine deaminase acting on RNA (ADAR1), a suppressor of double-stranded RNA-triggered innate immune responses. J Biol Chem. 2019;294:1710-20

50. Crow YJ, Chase DS, Lowenstein Schmidt J, Szynkiewicz M, Forte GM, Gornall HL, Oojageer A, Anderson B, Pizzino A, Helman G, et al. Characterization of human disease phenotypes associated with mutations in TREX1, RNASEH2A, RNASEH2B, RNASEH2C, SAMHD1, ADAR, and IFIH1. Am J Med Genet A. 2015;167A:296-312.

51. Mannion NM, Greenwood SM, Young R, Cox S, Brindle J, Read D, Nellåker C, Vesely C, Ponting CP, McLaughlin PJ, et al. The RNA-editing enzyme ADAR1 controls innate immune responses to RNA. Cell Rep. 2014;9:1482-94.

52. Rice Gl, Kasher PR, Forte GM, Mannion NM, Greenwood SM, Szynkiewicz M, Dickerson JE, Bhaskar SS, Zampini M, Briggs TA, et al. Mutations in ADAR1 cause Aicardi-Goutières syndrome associated with a type I interferon signature. Nat Genet. 2012;44:1243-8.

53. Liddicoat BJ, Piskol R, Chalk AM, Ramaswami G, Higuchi M, Hartner JC, Li JB, Seeburg PH, Walkley CR. RNA editing by ADAR1 prevents MDA5 sensing of endogenous dsRNA as nonself. Science. 2015;349:1115-20.

54. Pestal K, Funk CC, Snyder JM, Price ND, Treuting PM, Stetson DB. Isoforms of RNA-editing enzyme ADAR1 independently control nucleic acid sensor MDA5-driven autoimmunity and multi-organ development. Immunity. 2015;43:933-44.

55. Antonelli A, Ferrari SM, Corrado A, Di Domenicantonio A, Fallahi P. Autoimmune thyroid disorders. Autoimmun Rev. 2015;14:174-80.

56. Shiromoto Y, Sakurai M, Minakuchi M, Ariyoshi K, Nishikura K. ADAR1 RNA editing enzyme regulates R-loop formation and genome stability at telomeres in cancer cells. Nat Commun. 2021;12:1654.

57. Ramírez-Moya J, Baker AR, Slack FJ, Santisteban P. ADAR1-mediated RNA editing is a novel oncogenic process in thyroid cancer and regulates miR200 activity. Oncogene. 2020;39:3738-53.

58. Hsiao YE, Bahn JH, Yang Y, Lin X, Tran S, Yang EW, Quinones-Valdez G, Xiao $X$. RNA editing in nascent RNA affects pre-mRNA splicing. Genome Res. 2018;28:812-23.

59. Jeong S. SR proteins: binders, regulators, and connectors of RNA. Mol Cells. 2017;40:1-9.

60. Zhou Z, Fu XD. Regulation of splicing by SR proteins and SR proteinspecific kinases. Chromosoma. 2013;122:191-207.

61. Geuens T, Bouhy D, Timmerman V. The hnRNP family: insights into their role in health and disease. Hum Genet. 2016;135:851-67.

62. Coltri PP, Dos Santos MGP, da Silva GHG. Splicing and cancer: challenges and opportunities. Wiley Interdiscip Rev RNA. 2019;10:e1527. 
63. Sheth N, Roca X, Hastings ML, Roeder T, Krainer AR, Sachidanandam R. Comprehensive splice-site analysis using comparative genomics. Nucleic Acids Res. 2006;34:3955-67.

64. Zhang T, Kwiatkowski N, Olson CM, Dixon-Clarke SE, Abraham BJ, Greifenberg AK, Ficarro SB, Elkins JM, Liang Y, Hannett NM, et al. Covalent targeting of remote cysteine residues to develop CDK12 and CDK13 inhibitors. Nat Chem Biol. 2016;12:876-84.

65. Zeng M, Kwiatkowski NP, Zhang T, Nabet B, Xu M, Liang Y, Quan C, Wang J, Hao M, Palakurthi S, et al. Targeting MYC dependency in ovarian cancer through inhibition of CDK7 and CDK12/13. Elife. 2018;7:e39030.

66 Xu LD, Öhman M. ADAR1 editing and its role in cancer. Genes. 2018;10:12.

67. Dong X, Chen G, Cai Z, Li Z, Qiu L, Xu H, Yuan Y, Liu XL, Liu J. CDK13 RNA over-editing mediated by ADAR1 associates with poor prognosis of hepatocellular carcinoma patients. Cell Physiol Biochem. 2018;47:2602-12.

68. Sakurai M, Ueda H, Yano T, Okada S, Terajima H, Mitsuyama T, Toyoda A, Fujiyama A, Kawabata H, Suzuki T. A biochemical landscape of A-to-I RNA editing in the human brain transcriptome. Genome Res. 2014;24:522-34.

69. Kung CP, Cottrell KA, Ryu S, Bramel ER, Kladney RD, Bao EA, Freeman EC, Sabloak T, Maggi L, Weber JD. Evaluating the therapeutic potential of ADAR1 inhibition for triple-negative breast cancer. Oncogene. 2021;40:189-202.

70. Kuefer R, Day KC, Kleer CG, Sabel MS, Hofer MD, Varambally S, Zorn CS, Chinnaiyan AM, Rubin MA, Day ML. ADAM15 disintegrin is associated with aggressive prostate and breast cancer disease. Neoplasia. 2006:8:319-29.

71. Ortiz RM, Kärkkäinen I, Huovila AP. Aberrant alternative exon use and increased copy number of human metalloprotease-disintegrin ADAM15 gene in breast cancer cells. Genes Chromosomes Cancer. 2004;41:366-78.

72. Zhong JL, Poghosyan Z, Pennington CJ, Scott X, Handsley MM, Warn A, Gavrilovic J, Honert K, Krüger A, Span PN, et al. Distinct functions of natural ADAM-15 cytoplasmic domain variants in human mammary carcinoma. Mol Cancer Res. 2008;6:383-94.
73. Schackmann RC, Tenhagen M, van de Ven RA, Derksen PW. p120-catenin in cancer - mechanisms, models and opportunities for intervention. J Cell Sci. 2013;126:3515-25.

74. Cui J, Cai Y, Hu Y, Huang Z, Luo Y, Kaz AM, Yang Z, Chen D, Fan X, Grady WM, Wang J. Epigenetic silencing of TPM2 contributes to colorectal cancer progression upon RhoA activation. Tumour Biol. 2016;37:12477-83.

75. Zhang J, Xu S, Zhang X, Wang P, Wu H, Xia B, Zhang G, Lei B, Wan L, Zhang D, Pang D. Hypoxia-induced TPM2 methylation is associated with chemoresistance and poor prognosis in breast cancer. Cell Physiol Biochem. 2018;45:692-705

76. Mo YY, Reynolds AB. Identification of murine p120 isoforms and heterogeneous expression of p120cas isoforms in human tumor cell lines. Cancer Res. 1996;56:2633-40.

77. Assinder SJ, Au E, Dong Q, Winnick C. A novel splice variant of the beta-tropomyosin (TPM2) gene in prostate cancer. Mol Carcinog. 2010:49:525-31.

78. Zhang X, Zhuang R, Ye Q, Zhuo J, Chen K, Lu D, Wei X, Xie H, Xu X, Zheng $\mathrm{S}$. High expression of human AugminComplex submit 3 indicates poor prognosis and associates with tumor progression in hepatocellular carcinoma. J Cancer. 2019;10:1434-43.

\section{Publisher's Note}

Springer Nature remains neutral with regard to jurisdictional claims in published maps and institutional affiliations.
Ready to submit your research? Choose BMC and benefit from:

- fast, convenient online submission

- thorough peer review by experienced researchers in your field

- rapid publication on acceptance

- support for research data, including large and complex data types

- gold Open Access which fosters wider collaboration and increased citations

- maximum visibility for your research: over $100 \mathrm{M}$ website views per year

At BMC, research is always in progress.

Learn more biomedcentral.com/submissions 